Article

\title{
Duality in Fuzzy Sets and Dual Arithmetics of Fuzzy Sets
}

\author{
Hsien-Chung $\mathrm{Wu}(\mathbb{D}$ \\ Department of Mathematics, National Kaohsiung Normal University, Kaohsiung 802, Taiwan; \\ hcwu@nknucc.nknu.edu.tw
}

Received: 20 November 2018; Accepted: 20 December 2018; Published: 22 December 2018

\begin{abstract}
The conventional concept of $\alpha$-level sets of fuzzy sets will be treated as the upper $\alpha$-level sets. In this paper, the concept of lower $\alpha$-level sets of fuzzy sets will be introduced, which can also be regarded as a dual concept of upper $\alpha$-level sets of fuzzy sets. We shall also introduce the concept of dual fuzzy sets. Under these settings, we can establish the so-called dual decomposition theorem. We shall also study the dual arithmetics of fuzzy sets in $\mathbb{R}$ and establish some interesting results based on the upper and lower $\alpha$-level sets.
\end{abstract}

Keywords: dual decomposition theorem; dual fuzzy sets; dual arithmetics; lower $\alpha$-level sets; upper $\alpha$-level sets

\section{Introduction}

The properties of fuzzy numbers and the arithmetic of fuzzy quantities (or fuzzy numbers) have been studied for a long time. The interesting issue for studying the additive inverse of a fuzzy number may refer to Hong and Do [1], Vrba [2] and Wu [3,4]. Also, Anzilli and Facchinetti [5], Bodjanova [6], Dubois and Prade [7] investigated the median, mean and variance of fuzzy numbers. Wang et al. [8] studied the two-dimensional discrete fuzzy numbers. Mitchell and Schaefer [9] and Yager and Filev [10] studied the orderings of fuzzy numbers. On the other hand, Deschrijver [11,12] studied the arithmetic operators in interval-valued fuzzy set. Ban and Coroianu [13] investigated the approximation of fuzzy numbers. Guerra and Stefanini [14] studied the approximation of arithmetic of fuzzy numbers. Holčapek and Štěpnička [15] studied a new framework for arithmetics of extensional fuzzy numbers. Stupňanová [16] used a probabilistic approach to study the arithmetics of fuzzy numbers. Wu [17] used the decomposition and construction of fuzzy sets to study the arithmetic operations on fuzzy quantities. In this paper, we shall study the dual arithmetic of fuzzy sets by considering the dual membership function.

The $\alpha$-level set of a fuzzy set will be called the upper $\alpha$-level set. In this paper, we shall define the so-called lower $\alpha$-level set. The well-known (primal) decomposition theorem says that the membership function of a fuzzy set can be expressed in terms of the characteristic function of (upper) $\alpha$-level sets. In this paper, we are going to establish the so-called dual decomposition theorem by showing that the membership function can be expressed in terms of lower $\alpha$-level sets.

On the other hand, the concept of dual fuzzy set will be proposed by considering theso-called dual membership function. Based on the dual membership functions, we shall also study the so-called dual arithmetic of fuzzy sets in $\mathbb{R}$. The definition of arithmetic operations is based on the supremum and minimum of membership functions. Inspired by its form, we shall define the so-called dual arithmetic operations based on the infimum and maximum of dual membership functions. A duality relation is also established between the arithmetics and dual arithmetics.

In Section 2, we introduce the concept of lower $\alpha$-level sets and present some interesting properties that will be used in the subsequent discussion. In Section 3, we introduce the concept of dual fuzzy 
set and present some interesting results based on the lower $\alpha$-level sets. In Section 4 , we establish the so-called dual decomposition theorem. In Section 5, we introduce the dual arithmetics of fuzzy sets in $\mathbb{R}$ and establish some interesting results based on the upper and lower $\alpha$-level sets.

\section{Lower and Upper Level Sets}

Let $\tilde{A}$ be a fuzzy subset of a universal set $U$ with membership function denoted by $\tilde{\zeta}_{\tilde{A}}$. For $\alpha \in$ $(0,1]$, the $\alpha$-level set of $\tilde{A}$ is denoted and defined by

$$
\tilde{A}_{\alpha}=\left\{x \in U: \xi_{\tilde{A}}(x) \geq \alpha\right\} .
$$

For $\alpha \in[0,1)$, we also define

$$
\tilde{A}_{\alpha+}=\left\{x \in U: \xi_{\tilde{A}}(x)>\alpha\right\} .
$$

The support of a fuzzy set $\tilde{A}$ within a universal set $U$ is the crisp set defined by

$$
\tilde{A}_{0+}=\left\{x \in U: \xi_{\tilde{A}}(x)>0\right\} .
$$

The definition of 0-level set is an important issue in fuzzy sets theory. If the universal set $U$ is endowed with a topology $\tau$, then the 0-level set $\tilde{A}_{0}$ can be defined as the closure of the support of $\tilde{A}$, i.e.,

$$
\tilde{A}_{0}=\operatorname{cl}\left(\tilde{A}_{0+}\right) .
$$

If $U$ is not endowed with a topological structure, then the intuitive way for defining the 0-level set is to follow the equality (1) for $\alpha=0$. In this case, the 0 -level set of $\tilde{A}$ is the whole universal set $U$. This kind of 0-level set seems not so useful. Therefore, we always endow a topological structure to the universal set $U$ when the 0 -level set should be seriously considered.

Let $\tilde{A}$ be a fuzzy set in $U$ with membership function $\xi_{\tilde{A}}$. The range of $\xi_{\tilde{A}}$ is denoted by $\mathcal{R}\left(\xi_{\tilde{A}}\right)$ that is a subset of $[0,1]$. We see that the range $\mathcal{R}\left(\xi_{\tilde{A}}\right)$ can be a proper subset of $[0,1]$ with $\mathcal{R}\left(\xi_{\tilde{A}}\right) \neq[0,1]$. Define

$$
\alpha_{\tilde{A}}^{*}=\sup \mathcal{R}\left(\xi_{\tilde{A}}\right)=\sup _{x \in \tilde{A}_{0}} \xi_{\tilde{A}}(x) \text { and } \alpha_{\tilde{A}}^{\circ}=\inf \mathcal{R}\left(\xi_{\tilde{A}}\right)=\inf _{x \in \tilde{A}_{0}} \xi_{\tilde{A}}(x) .
$$

Remark 1. We have the following observations.

- $\quad$ For any $0 \leq \alpha \leq \alpha_{\tilde{A}^{\prime}}^{\circ}$, even though $\alpha \notin \mathcal{R}\left(\xi_{\tilde{A}}\right)$, we have $\tilde{A}_{\alpha}=\tilde{A}_{\alpha_{\tilde{A}}^{\circ}} \neq \varnothing$.

- $\quad$ For any $\alpha_{\tilde{A}}^{\circ}<\alpha<\alpha_{\tilde{A}^{\prime}}^{*}$, even though $\alpha \notin \mathcal{R}\left(\tilde{\xi}_{\tilde{A}}\right)$, we have $\tilde{A}_{\alpha} \neq \varnothing$. It is also obvious that $\tilde{A}_{\alpha}=\varnothing$ for $\alpha>\alpha_{\tilde{A}}^{*}$.

- If the maximum $\max \mathcal{R}\left(\xi_{\tilde{A}}\right)$ exists, i.e., $\sup \mathcal{R}\left(\xi_{\tilde{A}}\right)=\max \mathcal{R}\left(\xi_{\tilde{A}}\right)$, then we have $\tilde{A}_{\alpha_{\tilde{A}}^{*}} \neq \varnothing$. If $\max \mathcal{R}\left(\xi_{\tilde{A}}\right)$ does not exist, then $\tilde{A}_{\alpha_{\tilde{A}}^{*}}=\varnothing$.

Therefore we have the following interesting and useful result.

Proposition 1. Let $\tilde{A}$ be a fuzzy set in $U$ with membership function $\xi_{\tilde{A}}$. Define $\alpha_{\tilde{A}}^{*}=\sup \mathcal{R}\left(\xi_{\tilde{A}}\right)$ and

$$
I_{\tilde{A}}^{*}= \begin{cases}{\left[0, \alpha_{\tilde{A}}^{*}\right) \text { is a half-open interval, }} & \text { if the maximum } \max \mathcal{R}\left(\xi_{\tilde{A}}\right) \text { does not exist } \\ {\left[0, \alpha_{\tilde{A}}^{*}\right] \text { is a closed interval, }} & \text { if the maximum } \max \mathcal{R}\left(\xi_{\tilde{A}}\right) \text { exists. }\end{cases}
$$

Then $\tilde{A}_{\alpha} \neq \varnothing$ for all $\alpha \in I_{\tilde{A}}^{*}$ and $\tilde{A}_{\alpha}=\varnothing$ for all $\alpha \notin I_{\tilde{A}}^{*}$. Moreover, we have $\mathcal{R}\left(\xi_{\tilde{A}}\right) \subseteq I_{\tilde{A}}^{*}$. 
Remark 2. Recall that $\tilde{A}$ is called a normal fuzzy set in $U$ if and only if there exists $x \in U$ such that $\xi_{\tilde{A}}(x)=1$. In this case, we have $I_{\tilde{A}}^{*}=[0,1]$. However, the range $\mathcal{R}\left(\tilde{\zeta}_{\tilde{A}}\right)$ is not necessarily equal to $[0,1]$ even though $\tilde{A}$ is normal, since the membership function of $\tilde{A}$ is not necessarily a continuous function.

The 0 -level set $\tilde{A}_{0}$ of $\tilde{A}$ is also called the proper domain of $\tilde{A}$, since $\xi_{\tilde{A}}(x)=0$ for all $x \notin \tilde{A}_{0}$. For $\alpha \in I_{\tilde{A}}^{*}$, the $\alpha$-level set $\tilde{A}_{\alpha}$ of $\tilde{A}$ may be called the upper $\alpha$-level set (or upper $\alpha$-cut) of $\tilde{A}$. We also see that

$$
\tilde{A}_{\alpha}=\left\{x \in U: \xi_{\tilde{A}}(x) \geq \alpha\right\}=\left\{x \in \tilde{A}_{0}: \xi_{\tilde{A}}(x) \geq \alpha\right\}
$$

and

$$
\tilde{A}_{\alpha+}=\left\{x \in U: \xi_{\tilde{A}}(x)>\alpha\right\}=\left\{x \in \tilde{A}_{0}: \xi_{\tilde{A}}(x)>\alpha\right\}
$$

Next we shall consider the so-called lower $\alpha$-level set (or lower $\alpha$-cut) of $\tilde{A}$.

Definition 1. Let $\tilde{A}$ be a fuzzy set in a topological space $U$ with proper domain $\tilde{A}_{0}$. For $\alpha \in[0,1]$, the following set

$$
{ }_{\alpha} \tilde{A}=\left\{x \in \tilde{A}_{0}: \xi_{\tilde{A}}(x) \leq \alpha\right\}
$$

is called the lower $\alpha$-level set of $\tilde{A}$. For $\alpha \in(0,1]$, we also define

$$
{ }_{\alpha-} \tilde{A}=\left\{x \in \tilde{A}_{0}: \xi_{\tilde{A}}(x)<\alpha\right\} .
$$

We remark that the lower $\alpha$-level set ${ }_{\alpha} \tilde{A}$ is considered in the proper domain $\tilde{A}_{0}$ rather than the whole universal set $U$. In general, it is clear to see that

$$
{ }_{\alpha} \tilde{A}=\left\{x \in \tilde{A}_{0}: \xi_{\tilde{A}}(x) \leq \alpha\right\} \neq\left\{x \in U: \xi_{\tilde{A}}(x) \leq \alpha\right\} .
$$

Next, we present some interesting observations. We first recall that the notation $x \in A \backslash B$ means $x \in A$ and $x \notin B$.

Remark 3. Let $\tilde{A}$ be a fuzzy set in $U$ with range $\mathcal{R}\left(\xi_{\tilde{A}}\right)$. Recall the notations

$$
\alpha_{\tilde{A}}^{*}=\sup \mathcal{R}\left(\xi_{\tilde{A}}\right) \text { and } \alpha_{\tilde{A}}^{\circ}=\inf \mathcal{R}\left(\xi_{\tilde{A}}\right) .
$$

Then we have the following observations.

- $\quad$ For any $0 \leq \alpha<\alpha_{\tilde{A}^{\prime}}^{\circ}$ we have $\alpha \tilde{A}=\varnothing$. For any $\alpha>\alpha_{\tilde{A}^{\prime}}^{\circ}$ even though $\alpha \notin \mathcal{R}\left(\tilde{\zeta}_{\tilde{A}}\right)$, using $(4)$, we have

$$
{ }_{\alpha} \tilde{A}=\tilde{A}_{0} \backslash\left\{x \in \tilde{A}_{0}: \tilde{\zeta}_{\tilde{A}}(x)>\alpha\right\}=\tilde{A}_{0} \backslash\left\{x \in U: \xi_{\tilde{A}}(x)>\alpha\right\}=\tilde{A}_{0} \backslash \tilde{A}_{\alpha+} \neq \varnothing .
$$

It is also obvious that ${ }_{\alpha} \tilde{A}=\tilde{A}_{0}$ for $\alpha \geq \alpha_{\tilde{A}}^{*}$. If the minimum $\min \mathcal{R}\left(\xi_{\tilde{A}}\right)$ exists, i.e., $\inf \mathcal{R}\left(\xi_{\tilde{A}}\right)=$ $\min \mathcal{R}\left(\xi_{\tilde{A}}\right)$, then we have $\alpha_{\tilde{A}}^{\circ} \tilde{A} \neq \varnothing$ and if $\min \mathcal{R}\left(\xi_{\tilde{A}}\right)$ does not exist, then $\alpha_{\tilde{A}}^{\circ} \tilde{A}=\varnothing$.

- If $x \in{ }_{0} \tilde{A}$ then $x \in \tilde{A}_{0}$ and $\tilde{\xi}_{\tilde{A}}(x)=0$.

- For any $\alpha, \beta \in[0,1]$ with $\alpha<\beta$, we have ${ }_{\alpha} \tilde{A} \subseteq{ }_{\beta} \tilde{A}$.

- From Proposition 1, for $\alpha \in I_{\tilde{A}}^{*}$ with $\alpha>0$, we have

$$
\varnothing \neq \tilde{A}_{\alpha}=\left\{x \in \tilde{A}_{0}: \xi_{\tilde{A}}(x) \geq \alpha\right\}=\tilde{A}_{0} \backslash\left\{x \in \tilde{A}_{0}: \xi_{\tilde{A}}(x)<\alpha\right\}=\tilde{A}_{0} \backslash \alpha-\tilde{A} .
$$

Regarding the lower $\alpha$-level sets, from the first observation of Remark 3, we have the following interesting and useful result. 
Proposition 2. Let $\tilde{A}$ be a fuzzy set in $U$ with membership function $\xi_{\tilde{A}}$. Define $\alpha_{\tilde{A}}^{\circ}=\inf \mathcal{R}\left(\xi_{\tilde{A}}\right)$ and

$$
I_{\tilde{A}}^{\circ}= \begin{cases}\left(\alpha_{\tilde{A}}^{\circ}, 1\right] \text { is a half-open interval, } & \text { if the minimum } \min \mathcal{R}\left(\xi_{\tilde{A}}\right) \text { does not exist } \\ {\left[\alpha_{\tilde{A}}^{\circ}, 1\right] \text { is a closed interval, }} & \text { if the minimum } \min \mathcal{R}\left(\xi_{\tilde{A}}\right) \text { exists. }\end{cases}
$$

Then ${ }_{\alpha} \tilde{A} \neq \varnothing$ for all $\alpha \in I_{\tilde{A}}^{\circ}$ and ${ }_{\alpha} \tilde{A}=\varnothing$ for all $\alpha \notin I_{\tilde{A}}^{\circ}$.

Remark 4. If $\alpha_{\tilde{A}}^{\circ}=0$ in Proposition 2, then $I_{\tilde{A}}^{\circ}=[0,1]$. However, the range $\mathcal{R}\left(\tilde{\zeta}_{\tilde{A}}\right)$ is not necessarily equal to $[0,1]$ when $\tilde{A}$ is normal, since the membership function of $\tilde{A}$ is not necessarily a continuous function.

Based on the interval $I_{\tilde{A}}^{\circ}$ in Proposition 2, we present some basic properties of lower $\alpha$-level sets, which will be used in the further study.

Proposition 3. Let Ã be a fuzzy set in $U$. Then we have the following results.

(i) If $\alpha \in I_{\tilde{A}}^{\circ}$ with $\alpha<1$ and $\left\{\alpha_{n}\right\}_{n=1}^{\infty}$ is a decreasing sequence in $[0,1]$ such that $\alpha_{n} \downarrow \alpha$, then

$$
{ }_{\alpha} \tilde{A}=\bigcap_{n=1}^{\infty} \alpha_{n} \tilde{A} .
$$

(ii) For $\alpha \in I_{\tilde{A}}^{\circ}$ with $\alpha>0$, the following statements hold true.

- If $\alpha_{n} \uparrow \alpha$, then

$$
\bigcup_{n=1}^{\infty} \alpha_{n} \tilde{A} \subseteq{ }_{\alpha} \tilde{A} \text { and }{ }_{\alpha-} \tilde{A} \subseteq \bigcup_{n=1}^{\infty} \alpha_{n}-\tilde{A} \subseteq \bigcup_{n=1}^{\infty} \alpha_{n} \tilde{A}
$$

- If $\alpha_{n} \uparrow \alpha$ with $\alpha_{n}<\alpha$ for all $n$, then

$$
{ }_{\alpha-} \tilde{A}=\bigcup_{n=1}^{\infty} \alpha_{n}-\tilde{A}=\bigcup_{n=1}^{\infty} \alpha_{n} \tilde{A}
$$

(iii) If $\alpha \in I_{\tilde{A}}^{\circ}$ with $\alpha<1$, then

$$
{ }_{\alpha} \tilde{A}=\bigcap_{\alpha<\beta \leq 1} \beta \tilde{A}=\bigcap_{\alpha \leq \beta \leq 1}{ }_{\beta} \tilde{A} .
$$

(iv) If $\alpha \in I_{\tilde{A}}^{\circ}$ with $\alpha>0$, then

$$
\alpha-\tilde{A}=\bigcup_{0 \leq \beta<\alpha} \beta \tilde{A}
$$

Proof. To prove part (i), since $\alpha_{n} \geq \alpha$ for all $n=1,2, \cdots$, we have ${ }_{\alpha} \tilde{A} \subseteq \alpha_{n} \tilde{A}$ for all $n=1,2, \cdots$, which implies ${ }_{\alpha} \tilde{A} \subseteq \bigcap_{n=1}^{\infty} \alpha_{n} \tilde{A}$. On the other hand, for $x \in \bigcap_{n=1}^{\infty} \alpha_{n} \tilde{A}$, we have $\xi_{\tilde{A}}(x) \leq \alpha_{n}$ for all $n=1,2, \cdots$, which implies $\xi_{\tilde{A}}(x) \leq \inf _{n} \alpha_{n}=\alpha$. Therefore, we conclude that $x \in{ }_{\alpha} \tilde{A}$.

To prove part (ii), given $x \in{ }_{\alpha-} \tilde{A}$, we have $\xi_{\tilde{A}}(x)<\alpha$. Since $\alpha_{n} \uparrow \alpha$, given any $0<\epsilon \leq$ $\alpha-\xi_{\tilde{A}}(x)$, there exists $N$ such that $0<\alpha-\alpha_{N}<\epsilon$, which says that $\xi_{\tilde{A}}(x)<\alpha_{N}$, i.e., $x \in \bigcup_{n=1}^{\infty} \alpha_{n}-\tilde{A}$. Therefore, we obtain the inclusion $\alpha_{-} \tilde{A} \subseteq \bigcup_{n=1}^{\infty} \alpha_{n}-\tilde{A}$. On the other hand, since $\alpha_{n} \uparrow \alpha$, we have the following cases.

- If $\alpha_{n} \leq \alpha$ for all $n=1,2, \cdots$, then ${ }_{\alpha_{n}} \tilde{A} \subseteq{ }_{\alpha} \tilde{A}$, which implies $\bigcup_{n=1}^{\infty} \alpha_{n} \tilde{A} \subseteq{ }_{\alpha} \tilde{A}$.

- If $\alpha_{n}<\alpha$ for all $n=1,2, \cdots$, we see that $x \in \alpha_{n} \tilde{A}$ implies $\tilde{\xi}_{\tilde{A}}(x) \leq \alpha_{n}<\alpha$, which says that ${ }_{\alpha_{n}} \tilde{A} \subseteq{ }_{\alpha-} \tilde{A}$ for all $n=1,2, \cdots$. Therefore, we have $\bigcup_{n=1}^{\infty} \alpha_{n} \tilde{A} \subseteq{ }_{\alpha-} \tilde{A}$.

Then we obtain the desired equalities and inclusions.

To prove part (iii), for $1 \geq \beta>\alpha \geq 0$, we have ${ }_{\alpha} \tilde{A} \subseteq{ }_{\beta} \tilde{A}$. Therefore, we have the inclusion ${ }_{\alpha} \tilde{A} \subseteq \bigcap_{\beta \in(\alpha, 1]} \beta \tilde{A}$. On the other hand, given any $\epsilon>0$, for $x \in \bigcap_{\beta \in(\alpha, 1]} \tilde{A}$, we have $x \in{ }_{\alpha+\epsilon} \tilde{A}$, since 
$\alpha+\epsilon>\alpha$. This says that $\xi_{\tilde{A}}(x) \leq \alpha+\epsilon$, which also implies $\xi_{\tilde{A}}(x) \leq \alpha$, since $\epsilon$ is an arbitrary positive number (i.e., we can take $\epsilon \rightarrow 0$ ). Therefore, we conclude that $x \in{ }_{\alpha} \tilde{A}$.

To prove part (iv), for $\beta<\alpha$, if $x \in{ }_{\beta} \tilde{A}$, i.e., $\xi_{\tilde{A}}(x) \leq \beta<\alpha$, then $x \in{ }_{\alpha-} \tilde{A}$. Therefore we obtain ${ }_{\beta} \tilde{A} \subseteq{ }_{\alpha-} \tilde{A}$, which implies the inclusion $\bigcup_{0 \leq \beta<\alpha} \tilde{A} \subseteq{ }_{\alpha-} \tilde{A}$. On the other hand, given any $x \in{ }_{\alpha-} \tilde{A}$, we have $\xi_{\tilde{A}}(x)<\alpha$. By the denseness, there exists $\beta_{0}$ such that $\xi_{\tilde{A}}(x) \leq \beta_{0}<\alpha$, i.e., $x \in \bigcup_{0 \leq \beta<\alpha \beta} \tilde{A}$. Therefore, we obtain ${ }_{\alpha-} \tilde{A} \subseteq \bigcup_{0 \leq \beta<\alpha \beta} \tilde{A}$. This shows the desired equality, and the proof is complete.

Based on the interval $I_{\widetilde{A}}^{*}$ in Proposition 1, we can similarly obtain the following results.

Proposition 4. Let Ã be a fuzzy set in $U$. Then we have the following results.

(i) If $\alpha \in I_{\tilde{A}}^{*}$ with $\alpha>0$ and $\left\{\alpha_{n}\right\}_{n=1}^{\infty}$ is an increasing sequence in $[0,1]$ such that $\alpha_{n} \uparrow \alpha$, then

$$
\tilde{A}_{\alpha}=\bigcap_{n=1}^{\infty} \tilde{A}_{\alpha_{n}}
$$

(ii) For $\alpha \in I_{\tilde{A}}^{*}$ with $\alpha<1$, we have the following results.

- If $\alpha \in[0,1)$ and $\alpha_{n} \downarrow \alpha$, then $\bigcup_{n=1}^{\infty} \tilde{A}_{\alpha_{n}} \subseteq \tilde{A}_{\alpha}$ and $\tilde{A}_{\alpha+} \subseteq \bigcup_{n=1}^{\infty} \tilde{A}_{\alpha_{n}}$.

- If $\alpha \in[0,1)$ and $\alpha_{n} \downarrow \alpha$ with $\alpha_{n}>\alpha$ for all $n$, then $\tilde{A}_{\alpha+}=\bigcup_{n=1}^{\infty} \tilde{A}_{\alpha_{n}}$.

(iii) If $\alpha \in I_{\tilde{A}}^{*}$ with $\alpha>0$, then

$$
\tilde{A}_{\alpha}=\bigcap_{\beta \in[0, \alpha]} \tilde{A}_{\beta}=\bigcap_{\beta \in[0, \alpha)} \tilde{A}_{\beta} .
$$

(iv) If $\alpha \in I_{\tilde{A}}^{*}$ with $\alpha<1$, then

$$
\tilde{A}_{\alpha+}=\bigcup_{\alpha<\beta \leq 1} \tilde{A}_{\beta}
$$

Let $f: S \rightarrow \mathbb{R}$ be a real-valued function defined on a convex subset $S$ of a real vector space $U$. Recall that $f$ is quasi-convex on $S$ if and only if, for each $x, y \in S$, the following inequality is satisfied:

$$
f(\lambda x+(1-\lambda) y) \leq \max \{f(x), f(y)\}
$$

for each $0<\lambda<1$. It is well-known that $f$ is quasi-convex on $S$ if and only if the set $\{x \in S: f(x) \leq \alpha\}$ is convex for each $\alpha \in \mathbb{R}$. We also recall that $f$ is quasi-concave on $S$ if and only if $-f$ is quasi-convex on $S$. More precisely, the real-valued function $f$ is quasi-concave on $S$ if and only if

$$
f(\lambda x+(1-\lambda) y) \geq \min \{f(x), f(y)\}
$$

for each $0<\lambda<1$. We also have that $f$ is quasi-concave on $S$ if and only if the set $\{x \in S: f(x) \geq \alpha\}$ is convex for each $\alpha \in \mathbb{R}$.

Let $U$ be a vector space endowed with a topology, and let $\tilde{A}$ be a fuzzy subset of $U$ with membership function $\xi_{\tilde{A}}$. It is well-known that the membership function $\xi_{\tilde{A}}$ is quasi-concave if and only if the $\alpha$-level set $\tilde{A}_{\alpha}$ is a convex subset of $U$ for each $\alpha \in(0,1]$. In this case, the union $\bigcup_{0<\alpha \leq 1} \tilde{A}_{\alpha}$ is also a convex subset of $U$. This says that the upper zero-level set

$$
\tilde{A}_{0}=\mathrm{cl}\left(\bigcup_{0<\alpha \leq 1} \tilde{A}_{\alpha}\right)
$$

is a closed and convex subset of $U$. In particular, if $U=\mathbb{R}$ then the convex set $\tilde{A}_{\alpha}$ is reduced to be an interval for $\alpha \in[0,1]$.

Let $f:\left(U, \tau_{U}\right) \rightarrow \mathbb{R}$ be a real-valued function defined on a topological space $\left(U, \tau_{U}\right)$. Recall that $f$ is upper semi-continuous on $U$ if and only if $\{x \in U: f(x) \geq \lambda\}$ is a closed subset of $U$ for all $\lambda \in \mathbb{R}$, 
and $f$ is lower semi-continuous on $U$ if and only if $\{x \in U: f(x) \leq \lambda\}$ is a closed subset of $U$ for all $\lambda \in \mathbb{R}$. It is clear to see that if $f$ is upper semi-continuous on $U$ then $-f$ is lower semi-continuous on $U$, and if $f$ is lower semi-continuous on $U$ then $-f$ is upper semi-continuous on $U$.

Definition 2. Let $U$ be a vector space endowed with a topology, and let $\tilde{A}$ be a fuzzy subset of $U$ with membership function $\xi_{\tilde{A}}$. We denote by $\mathcal{F}_{c c}(U)$ the family of all fuzzy subsets of $U$ such that each $\tilde{A} \in \mathcal{F}_{c c}(U)$ satisfies the following conditions.

- $\quad$ The membership function $\xi_{\tilde{A}}$ is upper semi-continuous and quasi-concave on $U$.

- $\quad$ The upper 0-level set $\tilde{A}_{0}$ is a compact subset of $U$.

In particular, if $U=\mathbb{R}$ then each $\tilde{A} \in \mathcal{F}_{c c}(\mathbb{R})$ is called a fuzzy interval. If the fuzzy interval a is normal and the upper 1-level set $\tilde{a}_{1}$ is a singleton set $\{a\}$, where $a \in \mathbb{R}$, then $\tilde{a}$ is also called a fuzzy number with core value a. Usually, we write the upper case $\tilde{A}$ to denote the fuzzy interval, and write the lower case ã to denote the fuzzy number.

The upper semi-continuity and quasi-concavity says that each upper $\alpha$-level set $\tilde{A}_{\alpha}$ is a closed and convex subset of $U$ for $\alpha \in[0,1]$. Recall that, in a topological space, each closed subset of a compact set is a compact set. Since $\tilde{A}_{\alpha} \subseteq \tilde{A}_{0}$ for $\alpha \in(0,1]$, it follows that each upper $\alpha$-level set $\tilde{A}_{\alpha}$ is also a compact set for $\alpha \in(0,1]$.

Suppose that $\tilde{A}$ is a fuzzy interval. Then the upper 0-level set $\tilde{A}_{0}$ is a closed and bounded subset of $\mathbb{R}$. Also, the convexity, boundedness and closedness of each upper $\alpha$-level set $\tilde{A}_{\alpha}$ says that it is a bounded closed interval for $\alpha \in[0,1]$. More precisely, we have

$$
\tilde{A}_{\alpha}= \begin{cases}\varnothing & \text { if } \alpha \notin I_{\tilde{A}}^{*} \\ {\left[\tilde{A}_{\alpha}^{L}, \tilde{A}_{\alpha}^{U}\right]} & \text { if } \alpha \in I_{\tilde{A}}^{*}\end{cases}
$$

In particular, if $\tilde{a}$ is a fuzzy number, then $\tilde{a}_{\alpha}=\left[\tilde{a}_{\alpha}^{L}, \tilde{a}_{\alpha}^{U}\right]$ for all $\alpha \in[0,1]$ and $\tilde{a}_{1}^{L}=\tilde{a}_{1}^{U}$ for $\alpha=1$, i.e., the upper 1-level set $\tilde{a}_{1}=\left\{\tilde{a}_{1}^{L}=\tilde{a}_{1}^{U}=a\right\}$ is a singleton set, where $a$ is the core value.

Example 1. Let $\tilde{A}$ be a fuzzy interval. Then the upper $\alpha$-level set $\tilde{A}_{\alpha}$ is a closed interval given by $\tilde{A}_{\alpha}=\left[\tilde{A}_{\alpha}^{L}, \tilde{A}_{\alpha}^{U}\right]$ for all $\alpha \in[0,1]$. From part (iv) of Proposition 4 , for $\alpha \in I_{\tilde{A}}^{*}$ with $\alpha<1$, we have

$$
\tilde{A}_{\alpha+}=\bigcup_{\alpha<\beta \leq 1} \tilde{A}_{\beta}=\bigcup_{\alpha<\beta \leq 1}\left[\tilde{A}_{\beta}^{L}, \tilde{A}_{\beta}^{U}\right]
$$

Since $\tilde{A}_{\alpha} \subseteq \tilde{A}_{\beta}$ for $\beta<\alpha$, if we further assume that the end-points $\tilde{A}_{\alpha}^{L}$ and $\tilde{A}_{\alpha}^{U}$ are continuous functions with respect to $\alpha$ on $[0,1]$, then $\tilde{A}_{\alpha+}=\left(\tilde{A}_{\alpha}^{L}, \tilde{A}_{\alpha}^{U}\right)$ is an open interval. In this case, from Remark 3 , for $\alpha \in I_{\tilde{A}}^{\circ}$ with $\alpha>\alpha_{\tilde{A}^{\prime}}^{\circ}$ the lower $\alpha$-level set $\alpha \tilde{A}$ is given by

$$
{ }_{\alpha} \tilde{A}=\tilde{A}_{0} \backslash \tilde{A}_{\alpha+}=\left[\tilde{A}_{0}^{L}, \tilde{A}_{0}^{U}\right] \backslash\left(\tilde{A}_{\alpha}^{L}, \tilde{A}_{\alpha}^{U}\right)=\left[\tilde{A}_{0}^{L}, \tilde{A}_{\alpha}^{L}\right] \cup\left[\tilde{A}_{\alpha}^{U}, \tilde{A}_{0}^{U}\right]
$$

that is also a closed set in $\mathbb{R}$, where $\alpha_{\tilde{A}}^{\circ}=\inf \mathcal{R}\left(\xi_{\tilde{A}}\right)$. This also says that the membership function $\xi_{\tilde{A}}$ of $\tilde{A}$ is lower semi-continuous. Therefore we conclude that the membership function of $\tilde{A}$ is continuous. We also see that the lower 1-level set is

$$
{ }_{1} \tilde{A}=\tilde{A}_{0}=\left[\tilde{A}_{0}^{L}, \tilde{A}_{0}^{U}\right] .
$$

Suppose that the minimum $\min \mathcal{R}\left(\xi_{\tilde{A}}\right)$ exists, i.e.,

$$
\alpha_{\tilde{A}}^{\circ}=\inf \mathcal{R}\left(\xi_{\tilde{A}}\right)=\min \mathcal{R}\left(\xi_{\tilde{A}}\right)
$$


Then the lower $\alpha_{\tilde{A}}^{\circ}$-level set of $\tilde{A}$ consists of two points as

$$
\alpha_{\tilde{A}}^{\circ} \tilde{A}=\left\{\tilde{A}_{\alpha_{\tilde{A}}^{\circ}}^{L}, \tilde{A}_{\alpha_{\tilde{A}}^{\circ}}^{U}\right\}
$$

We also see that ${ }_{\alpha} \tilde{A}=\varnothing$ for $0 \leq \alpha<\alpha_{\tilde{A}}^{\circ}$.

\section{Dual Fuzzy Sets}

Let $\tilde{A}$ be a fuzzy set in $U$ with membership function $\xi_{\tilde{A}}$ defined on $U$. Recall that the membership function of complement fuzzy set of $\tilde{A}$ is denoted and defined by $\xi_{\tilde{A}^{c}}=1-\xi_{\tilde{A}}$ that is also defined on $U$. Since the 0 -level set $\tilde{A}_{0}$ is treated as the proper domain of $\tilde{A}$, we consider the restrict function $\left.\xi_{\tilde{A}^{c}}\right|_{\tilde{A}_{0}}$ of $\xi_{\tilde{A}^{c}}$ on $\tilde{A}_{0}$ and define a function $\xi_{\tilde{A}^{\star}}: \tilde{A}_{0} \rightarrow[0,1]$ on $\tilde{A}_{0}$ by

$$
\xi_{\tilde{A}^{\star}}(x)=\left.\xi_{\tilde{A}^{c}}\right|_{\tilde{A}_{0}}(x)=1-\xi_{\tilde{A}}(x) .
$$

Then we use the notation $\tilde{A}^{\star}$ to denote the dual fuzzy set of $\tilde{A}$. The membership function of $\tilde{A}^{\star}$ is given in (6) that is also called a dual membership function. We also remark that $\xi_{\tilde{A}^{\star}} \neq \xi_{\tilde{A}^{c}}$, since their domains are different.

Remark 5. The relationships between the $\alpha$-level sets of $\tilde{A}$ and $\tilde{A}^{\star}$ are given below

- If $\tilde{A}_{\alpha} \neq \varnothing$ and ${ }_{1-\alpha} \tilde{A}^{\star} \neq \varnothing$, then

$$
\tilde{A}_{\alpha}=\left\{x \in U: \xi_{\tilde{A}}(x) \geq \alpha\right\}=\left\{x \in U: \tilde{\zeta}_{\tilde{A}^{\star}}(x) \leq 1-\alpha\right\}={ }_{1-\alpha} \tilde{A}^{\star}
$$

- $\quad$ If ${ }_{\alpha} \tilde{A} \neq \varnothing$ and $\tilde{A}_{1-\alpha}^{\star} \neq \varnothing$, then

$$
{ }_{\alpha} \tilde{A}=\left\{x \in U: \xi_{\tilde{A}}(x) \leq \alpha\right\}=\left\{x \in U: \xi_{\tilde{A}^{\star}}(x) \geq 1-\alpha\right\}=\tilde{A}_{1-\alpha}^{\star}
$$

- If $\tilde{A}_{\alpha}^{\star} \neq \varnothing$ and ${ }_{1-\alpha} \tilde{A} \neq \varnothing$, then

$$
\tilde{A}_{\alpha}^{\star}=\left\{x \in U: \tilde{\zeta}_{\tilde{A}^{\star}}(x) \geq \alpha\right\}=\left\{x \in U: \xi_{\tilde{A}}(x) \leq 1-\alpha\right\}={ }_{1-\alpha} \tilde{A}
$$

- $\quad$ If ${ }_{\alpha} \tilde{A}^{\star} \neq \varnothing$ and $\tilde{A}_{1-\alpha} \neq \varnothing$, then

$$
{ }_{\alpha} \tilde{A}^{\star}=\left\{x \in U: \xi_{\tilde{A}^{\star}}(x) \leq \alpha\right\}=\left\{x \in U: \xi_{\tilde{A}}(x) \geq 1-\alpha\right\}=\tilde{A}_{1-\alpha} .
$$

Let us recall that notation $I_{\tilde{A}}^{*}$ in Proposition 1 and the notation $I_{\tilde{A}}^{\circ}$ in Proposition 2 . Then we have the following interesting results.

Proposition 5. Let $\tilde{A}$ be a fuzzy set in $U$ with the dual fuzzy set $\tilde{A}^{\star}$. For $\alpha, \beta \in[0,1]$ with $\alpha+\beta=1$, we have the following properties.

(i) $\alpha \in I_{\tilde{A}}^{*}$ if and only if $\beta \in I_{\tilde{A}^{\star}}^{\circ}$.

(ii) If $\alpha \in I_{\tilde{A}}^{*}$ or $\beta \in I_{\tilde{A}^{\star}}^{\circ}$ then $\tilde{A}_{\alpha}={ }_{\beta} \tilde{A}^{\star}$.

Proof. We first have

$$
1-\alpha_{\tilde{A}}^{*}=1-\sup _{x \in \tilde{A}_{0}} \xi_{\tilde{A}}(x)=1+\inf _{x \in \tilde{A}_{0}}\left(-\xi_{\tilde{A}}(x)\right)=\inf _{x \in \tilde{A}_{0}}\left(1-\xi_{\tilde{A}}(x)\right)=\inf _{x \in \tilde{A}_{0}} \tilde{\zeta}_{\tilde{A}^{\star}}(x)=\alpha_{\tilde{A}^{\star}}^{\circ}
$$

The equalities (7) also say that the maximum $\max \mathcal{R}\left(\xi_{\tilde{A}}\right)$ exists if and only if the minimum $\min \mathcal{R}\left(\xi_{\tilde{A}^{\star}}\right)$ exists. To prove part (i), suppose that $\alpha \in I_{\tilde{A}}^{*}$. We have two cases. 
- $\quad$ Assume that the maximum $\max \mathcal{R}\left(\xi_{\tilde{A}}\right)$ exists. Then the minimum $\min \mathcal{R}\left(\xi_{\tilde{A}^{\star}}\right)$ exists and $0 \leq \alpha \leq \alpha_{\tilde{A}^{\prime}}^{*}$, Therefore we obtain $1 \geq \beta \geq 1-\alpha_{\tilde{A}^{\prime}}^{*}$ which also says that $1 \geq \beta \geq \alpha_{\tilde{A}}^{\circ}$ by using (7). This shows that $\beta \in I_{\tilde{A}^{\star}}^{\circ}$.

- $\quad$ Assume that the maximum $\max \mathcal{R}\left(\xi_{\tilde{A}}\right)$ doe not exist. Then the minimum $\min \mathcal{R}\left(\xi_{\tilde{A}^{\star}}\right)$ does not exist and $0 \leq \alpha<\alpha_{\tilde{A}}^{*}$. Therefore we obtain $1 \geq \beta>1-\alpha_{\tilde{A}}^{*}$, which also says that $1 \geq \beta>\alpha_{\tilde{A}}^{\circ}$ by using (7). This shows that $\beta \in I_{\tilde{A}^{\star}}^{\circ}$.

For the converse, suppose that $\beta \in I_{\tilde{A}^{\star}}^{\circ}$. Then we can similarly show that $\alpha \in I_{\tilde{A}}^{*}$. Part (ii) follows from Remark 5 and part (i) immediately. This completes the proof.

Let $\tilde{A} \in \mathcal{F}_{c c}(U)$. Then the membership function $\xi_{\tilde{A}}$ is upper semi-continuous and quasi-concave on $\tilde{A}_{0}$. It is also clear to see that the membership function $\tilde{\xi}_{\tilde{A}^{\star}}=1-\xi_{\tilde{A}}$ of dual fuzzy set $\tilde{A}^{\star}$ is lower semi-continuous and quasi-convex on $\tilde{A}_{0}$. This says that the lower $\alpha$-level set ${ }_{\alpha} \tilde{A}^{\star}$ is a closed and convex subset of $\tilde{A}_{0}$ for $\alpha \in(0,1]$.

Example 2. Let $\tilde{A}$ be a fuzzy interval with dual fuzzy set $\tilde{A}^{\star}$. Since the upper 0-level set $\tilde{A}_{0}$ is bounded and the lower $\alpha$-level set ${ }_{\alpha} \tilde{A}^{\star}$ is a closed and convex subset of $\mathbb{R}$ for $\alpha \in(0,1]$, it follows that ${ }_{\alpha} \tilde{A}^{\star}$ is also a bounded closed interval given by

$$
{ }_{\alpha} \tilde{A}^{\star}= \begin{cases}\varnothing & \text { if } \alpha \notin I_{\tilde{\tilde{A}}^{\star}}^{\circ} \\ \left.{ }_{\alpha} \tilde{A}^{\star L},{ }_{\alpha} \tilde{A}^{\star U}\right] & \text { if } \alpha \in I_{\tilde{A}^{\star \prime}}^{\circ}\end{cases}
$$

Using part (ii) of Proposition 5, we also have

$$
{ }_{\alpha} \tilde{A}^{\star}=\left\{\begin{array}{ll}
\varnothing & \text { if } \alpha \notin I_{\tilde{A}^{\star}}^{\circ} \\
\tilde{A}_{1-\alpha} & \text { if } \alpha \in I_{\tilde{A}^{\star}}^{\circ}
\end{array}= \begin{cases}\varnothing & \text { if } \alpha \notin I_{\tilde{A}^{\star}}^{\circ} \\
{\left[\tilde{A}_{1-\alpha^{\prime}}^{L}, \tilde{A}_{1-\alpha}^{U}\right]} & \text { if } \alpha \in I_{\tilde{A}^{\star}}^{\circ}\end{cases}\right.
$$

This shows that

$$
{ }_{\alpha} \tilde{A}^{\star L}=\tilde{A}_{1-\alpha}^{L} \text { and }{ }_{\alpha} \tilde{A}^{\star U}=\tilde{A}_{1-\alpha}^{U} .
$$

From part (iv) of Proposition 3, for $\alpha \in I_{\tilde{A}^{\star}}^{\circ}$ with $\alpha>0$, we have

$$
{ }_{\alpha-} \tilde{A}^{\star}=\bigcup_{0 \leq \beta<\alpha} \beta \tilde{A}^{\star}=\bigcup_{0 \leq \beta<\alpha}\left[\beta \tilde{A}^{\star L},{ }_{\beta} \tilde{A}^{\star U}\right] .
$$

Since ${ }_{\beta} \tilde{A}^{\star} \subseteq{ }_{\alpha} \tilde{A}^{\star}$ for $\beta<\alpha$, if we further assume that the end-points ${ }_{\beta} \tilde{A}^{\star L}$ and ${ }_{\beta} \tilde{A}^{\star U}$ are continuous functions with respect to $\beta$ on $[0,1]$, then ${ }_{\alpha-} \tilde{A}^{\star}=\left({ }_{\alpha} \tilde{A}^{\star L},{ }_{\alpha} \tilde{A}^{\star U}\right)$ is an open interval. In this case, from Remark 3, for $\alpha \in I_{\tilde{A}^{\star}}^{*}$ with $\alpha>0$, the upper $\alpha$-level set $\tilde{A}_{\alpha}^{\star}$ is given by

$$
\tilde{A}_{\alpha}^{\star}=\tilde{A}_{0}^{\star} \backslash{ }_{\alpha}-\tilde{A}^{\star}=\left[\tilde{A}_{0}^{\star L}, \tilde{A}_{0}^{\star U}\right] \backslash\left({ }_{\alpha} \tilde{A}^{\star L},{ }_{\alpha} \tilde{A}^{\star U}\right)=\left[\tilde{A}_{0}^{L},{ }_{\alpha} \tilde{A}^{\star L}\right] \cup\left[{ }_{\alpha} \tilde{A}^{\star U}, \tilde{A}_{0}^{U}\right]
$$

that is a closed set in $\mathbb{R}$. This also says that the membership function of $\tilde{A}^{\star}$ is upper semi-continuous. Therefore, we conclude that the membership functions of $\tilde{A}^{\star}$ and $\tilde{A}$ are continuous.

\section{Dual Decomposition Theorems}

Let $A$ be a subset of $U$. The characteristic function $\chi_{A}$ of $A$ is defined to be

$$
\chi_{A}(x)= \begin{cases}1 & \text { if } x \in A \\ 0 & \text { if } x \notin A\end{cases}
$$

Now we define the so-called dual characteristic function $\chi_{A}^{\star}$ of $A$ as follows

$$
\chi_{A}^{\star}(x)= \begin{cases}0 & \text { if } x \in A \\ 1 & \text { if } x \notin A .\end{cases}
$$


It is clear to see that

$$
\chi_{A}(x)+\chi_{A}^{\star}(x)=1 \text { for each } x \in U \text {. }
$$

Let $\tilde{A}$ be a normal fuzzy set in $U$. The well-known (primal) decomposition theorem says that the membership function $\xi_{\tilde{A}}$ can be expressed as

$$
\xi_{\tilde{A}}(x)=\sup _{\alpha \in[0,1]} \alpha \cdot \chi_{\tilde{A}_{\alpha}}(x)=\sup _{\alpha \in(0,1]} \alpha \cdot \chi_{\tilde{A}_{\alpha}}(x)
$$

where $\chi_{\tilde{A}_{\alpha}}$ is the characteristic function of the $\alpha$-level set $\tilde{A}_{\alpha}$. If $\tilde{A}$ is not normal, then we can also show that

$$
\xi_{\tilde{A}}(x)=\sup _{\alpha \in I_{\tilde{A}}^{*}} \alpha \cdot \chi_{\tilde{A}_{\alpha}}(x)=\sup _{\alpha \in \mathcal{R}\left(\xi_{\tilde{A}}\right)} \alpha \cdot \chi_{\tilde{A}_{\alpha}}(x) .
$$

The (primal) decomposition theorem says that the membership function can be expressed in terms of upper $\alpha$-level sets. In the sequel, we are going to show that the membership function can also be expressed in terms of lower $\alpha$-level sets as the following form

$$
\xi_{\tilde{A}}(x)=\sup _{\alpha \in I_{\tilde{A}}^{\circ}} \alpha \cdot \chi_{\alpha \tilde{A}}^{\star}(x)=\sup _{\alpha \in \mathcal{R}\left(\xi_{\tilde{A}}\right)} \alpha \cdot \chi_{\alpha \tilde{A}}^{\star}(x)
$$

where $\chi_{\alpha}^{\star} \tilde{A}$ is the dual characteristic function of lower $\alpha$-level set ${ }_{\alpha} \tilde{A}$.

Proposition 6. Let $\tilde{A}$ be a fuzzy set in a vector space $U$ that is also endowed with a topology. Given any fixed $x \in U$, we have the following results.

(i) Suppose that the minimum $\min \mathcal{R}\left(\xi_{\tilde{A}}\right)$ exists. Then the function $\zeta_{x}(\alpha)=\alpha \cdot \chi_{\alpha} \tilde{A}(x)$ is lower semi-continuous on $I_{\tilde{A}}^{\circ}$.

(ii) Suppose that the maximum $\max \mathcal{R}\left(\xi_{\tilde{A}}\right)$ exists. Then the function $\eta_{x}(\alpha)=\alpha \cdot \chi_{\tilde{A}_{\alpha}}(x)$ is upper semi-continuous on $I_{\tilde{A}}^{*}$.

Proof. To prove part (i), from Proposition 2, we see that $I_{\tilde{A}}^{\circ}$ is a closed interval. We need to show that the following set

$$
F_{r}=\left\{\alpha \in I_{\tilde{A}}^{\circ}: \zeta_{x}(\alpha) \leq r\right\}=\left\{\alpha \in I_{\tilde{A}}^{\circ}: \alpha \cdot \chi_{\alpha} \tilde{A}(x) \leq r\right\}
$$

is closed for each $r \in \mathbb{R}$. If $r<0$ then $F_{r}=\varnothing$ is closed. If $r=0$ then $F_{r}=\{0\}$ a singleton set is closed. If $r \geq 1$ then $F_{r}=I_{\tilde{A}}^{\circ}$ is also closed. Therefore we remain to show that $F_{r}$ is closed for each $r \in(0,1)$. Now, for each $\alpha \in \operatorname{cl}\left(F_{r}\right)$, there exists a sequence $\left\{\alpha_{n}\right\}_{n=1}^{\infty}$ in $F_{r}$ such that $\alpha_{n} \rightarrow \alpha$, i.e., $\alpha_{n} \leq r$ and $x \in \alpha_{n} \tilde{A}$ for all $n$. Then we have

$$
\alpha=\lim _{n \rightarrow \infty} \alpha_{n} \leq r<1 .
$$

We also see that there exists a subsequence $\left\{\alpha_{n_{k}}\right\}_{k=1}^{\infty}$ of $\left\{\alpha_{n}\right\}_{n=1}^{\infty}$ such that $\alpha_{n_{k}} \downarrow \alpha$ or $\alpha_{n_{k}} \uparrow \alpha$.

- $\quad$ Suppose that $\alpha_{n_{k}} \uparrow \alpha$, i.e., $\alpha \geq \alpha_{n_{k}}$ for all $k$. Then we have $x \in{ }_{\alpha} \tilde{A}$, since $\alpha_{n_{k}} \tilde{A} \subseteq \alpha \tilde{A}$ for all $k$. This says that $\alpha \in F_{r}$, since $\alpha \leq r$.

- $\quad$ Suppose that $\alpha_{n_{k}} \downarrow \alpha$. Since $\bar{x} \in{ }_{\alpha_{n_{k}}} \tilde{A}$ for all $k$, using part (i) of Proposition 3, we have $x \in{ }_{\alpha} \tilde{A}$. This says that $\alpha \in F_{r}$, since $\alpha \leq r$.

Therefore, we conclude that $\mathrm{cl}\left(F_{r}\right) \subseteq F_{r}$, i.e., $F_{r}$ is closed.

To prove part (ii), from Proposition 1 , we see that $I_{\tilde{A}}^{*}$ is a closed interval. We need to show that the following set

$$
F_{r}=\left\{\alpha \in I_{\tilde{A}}^{*}: \eta_{x}(\alpha) \geq r\right\}=\left\{\alpha \in I_{\tilde{A}}^{*}: \alpha \cdot \chi_{\tilde{A}_{\alpha}}(x) \geq r\right\}
$$

is closed for each $r \in \mathbb{R}$. If $r \leq 0$ then $F_{r}=I_{\tilde{A}}^{*}$ is closed. If $r>1$ then $F_{r}=\varnothing$ is closed. If $r=1$ then $F_{r}=\varnothing$ or $F_{r}=\{1\}$ a singleton set is also closed. Therefore we remain to show that $F_{r}$ is closed for 
each $r \in(0,1)$. Now, for each $\alpha \in \operatorname{cl}\left(F_{r}\right)$, since $r>0$, we have $\alpha>0$. Therefore, there exists a sequence $\left\{\alpha_{n}\right\}_{n=1}^{\infty}$ such that $\alpha_{n} \rightarrow \alpha$ and $\alpha_{n} \in F_{r}$ for all $n$, i.e., $\alpha_{n} \geq r$ and $x \in \tilde{A}_{\alpha_{n}}$ for all $n$. Then we have $\alpha \geq r$. We also see that there exists a subsequence $\left\{\alpha_{n_{k}}\right\}_{k=1}^{\infty}$ of $\left\{\alpha_{n}\right\}_{n=1}^{\infty}$ such that $\alpha_{n_{k}} \downarrow \alpha$ or $\alpha_{n_{k}} \uparrow \alpha$.

- $\quad$ If $\alpha_{n_{k}} \downarrow \alpha$, i.e., $\alpha \leq \alpha_{n_{k}}$ for all $k$, then $x \in \tilde{A}_{\alpha}$, since $\tilde{A}_{\alpha_{n_{k}}} \subseteq \tilde{A}_{\alpha}$ for all $k$ by part (i) of Proposition 4 . This says that $\alpha \in F_{r}$, since $\alpha \geq r$.

- If $\alpha_{n_{k}} \uparrow \alpha$, Then since $x \in \tilde{A}_{\alpha_{n_{k}}}$ for all $k$ by part (ii) of Proposition 4 , we have $x \in \tilde{A}_{\alpha}$. This says that $\alpha \in F_{r}$, since $\alpha \geq r$.

Therefore, we conclude that $\mathrm{cl}\left(F_{r}\right) \subseteq F_{r}$, i.e., $F_{r}$ is closed. This completes the proof.

Theorem 1. (Dual Decomposition Theorem) Let $\tilde{A}$ be a fuzzy set in $U$ with proper domain $\tilde{A}_{0}$. For $x \in \tilde{A}_{0}$, the membership degree $\xi_{\tilde{A}}(x)$ can be expressed in terms of lower $\alpha$-level sets as follows

$$
\begin{aligned}
\xi_{\tilde{A}}(x) & =\sup _{\alpha \in \mathcal{R}\left(\xi_{\tilde{A}}\right)} \alpha \cdot \chi_{\alpha \tilde{A}}^{\star}(x)=\max _{\alpha \in \mathcal{R}\left(\tilde{\zeta}_{\tilde{A}}\right)} \alpha \cdot \chi_{\alpha \tilde{A}}^{\star}(x)=\max _{\alpha \in \mathcal{R}\left(\tilde{\xi}_{\tilde{A}}\right)} \alpha \cdot\left[1-\chi_{\alpha} \tilde{A}(x)\right] \\
& =\sup _{\alpha \in I_{\tilde{A}}^{\circ}} \alpha \cdot \chi_{\alpha}^{\star}(x)=\max _{\alpha \in I_{\tilde{A}}^{\circ}} \alpha \cdot \chi_{\alpha \tilde{A}}^{\star}(x)=\max _{\alpha \in I_{\tilde{A}}^{\circ}} \alpha \cdot\left[1-\chi_{\alpha \tilde{A}}(x)\right],
\end{aligned}
$$

where $I_{\tilde{A}}^{\circ}$ is given in (5).

Proof. Let $\alpha_{0}=\xi_{\tilde{A}}(x) \in \mathcal{R}\left(\xi_{\tilde{A}}\right) \subseteq I_{\tilde{A}}^{\circ}$, i.e., $\alpha_{0} \in I_{\tilde{A}}^{\circ}$. Suppose that $\alpha_{0}=0$. If $x \notin{ }_{\alpha} \tilde{A} \neq \varnothing$ for some $\alpha \in I_{\tilde{A}}^{\circ}$, then $\xi_{\tilde{A}}(x)>\alpha \geq 0$, which contradicts $\xi_{\tilde{A}}(x)=\alpha_{0}=0$. Therefore $x \in{ }_{\alpha} \tilde{A}$ for all $\alpha \in I_{\tilde{A}}^{\circ}$, which says that $\alpha \cdot \chi_{\alpha \tilde{A}}^{\star}(x)=0$ for all $\alpha \in I_{\tilde{A}}^{\circ}$. This shows that the equalities in (8) are satisfied. Now we assume $\alpha_{0}>0$. Then $x \in \alpha_{0} \tilde{A}$. For $\alpha \in I_{\tilde{A}}^{\circ}$ with $\alpha<\alpha_{0}$, if $x \in{ }_{\alpha} \tilde{A}$, then $\xi_{\tilde{A}}(x) \leq \alpha<\alpha_{0}$, which contradicts $\alpha_{0}=\xi_{\tilde{A}}(x)$. Therefore, we have $x \notin \alpha \tilde{A}$ for $\alpha \in I_{\tilde{A}}^{\circ}$ with $\alpha<\alpha_{0}$. If $\alpha \in I_{\tilde{A}}^{\circ}$ with $\alpha \geq \alpha_{0}$, then $x \in \alpha_{0} \tilde{A} \subseteq{ }_{\alpha} \tilde{A}$, which says that $x \in{ }_{\alpha} \tilde{A}$ for $\alpha \geq \alpha_{0}$. Then we obtain

$$
\begin{aligned}
\sup _{\alpha \in I_{\tilde{A}}^{\circ}} \alpha \cdot \chi_{\alpha}^{\star} \tilde{A}(x) & =\max \left\{\sup _{\left\{\alpha \in I_{\tilde{A}}^{\circ}: \alpha<\alpha_{0}\right\}} \alpha \cdot \chi_{\alpha}^{\star} \tilde{A}(x), \sup _{\left\{\alpha \in I_{\tilde{A}}^{\circ}: \alpha \geq \alpha_{0}\right\}} \alpha \cdot \chi_{\alpha}^{\star} \tilde{A}(x)\right\} \\
& =\max \left\{\sup _{\left\{\alpha \in I_{\tilde{A}}^{\circ}: \alpha<\alpha_{0}\right\}} \alpha, 0\right\}=\max \left\{\alpha_{0}, 0\right\}=\alpha_{0}=\xi_{\tilde{A}}(x) .
\end{aligned}
$$

Since $\alpha_{0} \in I_{\tilde{A}^{\prime}}^{\circ}$ the above supremum is attained. It means that

$$
\xi_{\tilde{A}}(x)=\max _{\alpha \in I_{\tilde{A}}^{\circ}} \alpha \cdot \chi_{\alpha}^{\star} \tilde{A}(x) .
$$

The above arguments are still valid when $I_{\tilde{A}}^{\circ}$ is replaced by $\mathcal{R}\left(\xi_{\tilde{A}}\right)$. Therefore we obtain the desired equalities. This completes the proof.

Remark 6. The decomposition theorem for dual fuzzy set $\tilde{A}^{\star}$ based on the upper $\alpha$-level sets of $\tilde{A}^{\star}$ is given by

$$
\xi_{\tilde{A}^{\star}}(x)=\sup _{\alpha \in \mathcal{R}\left(\tilde{\zeta}_{\tilde{A}^{\star}}\right)} \alpha \cdot \chi_{\tilde{A}_{\alpha}^{\star}}(x) .
$$

According to Theorem 1, the dual decomposition theorem for $\tilde{A}^{\star}$ based on the lower $\alpha$-level sets of $\tilde{A}^{\star}$ is given by 


$$
\begin{aligned}
\xi_{\tilde{A}^{\star}}(x) & =\sup _{\alpha \in \mathcal{R}\left(\tilde{\mathcal{S}}_{\tilde{A}^{\star}}\right)} \alpha \cdot \chi_{\alpha}^{\star} \tilde{A}^{\star}(x)=\max _{\alpha \in \mathcal{R}\left(\tilde{\zeta}_{\tilde{A}^{\star}}\right)} \alpha \cdot \chi_{\alpha}^{\star} \tilde{A}^{\star}(x)=\max _{\alpha \in \mathcal{R}\left(\tilde{H}_{\tilde{A}^{\star}}\right)} \alpha \cdot\left[1-\chi_{\alpha} \tilde{A}^{\star}(x)\right] \\
& =\sup _{\alpha \in I_{\tilde{A}^{\star}}^{\circ}} \alpha \cdot \chi_{\alpha}^{\star} \tilde{A}^{\star}(x)=\max _{\alpha \in I_{\tilde{A}^{\star}}} \alpha \cdot \chi_{\alpha}^{\star} \tilde{A}^{\star}(x)=\max _{\alpha \in I_{\tilde{A}^{\star}}} \alpha \cdot\left[1-\chi_{\alpha} \tilde{A}^{\star}(x)\right] .
\end{aligned}
$$

Next we are going to present the dual decomposition theorem on a countable set. We write $\mathbb{Q}(0,1]=\mathbb{Q} \cap(0,1]$, where $\mathbb{Q}$ denotes the set of all rational numbers. It well-known that the countable set $\mathbb{Q}$ is dense in $\mathbb{R}$. This means that, given any $r \in \mathbb{R}$, there exist two sequences $\left\{p_{n}\right\}_{n=1}^{\infty}$ and $\left\{q_{n}\right\}_{n=1}^{\infty}$ in the countable set $\mathbb{Q}$ such that $p_{n} \uparrow r$ and $q_{n} \downarrow r$ as $n \rightarrow \infty$.

Theorem 2. (Dual Decomposition Theorem) Let $\tilde{A}$ be a fuzzy set in $U$ with proper domain $\tilde{A}_{0}$. Suppose that $\inf \mathcal{R}\left(\xi_{\tilde{A}}\right)=0$. For $x \in \tilde{A}_{0}$, the membership degree $\xi_{\tilde{A}}(x)$ can be expressed in terms of lower $\alpha$-level sets as follows

$$
\begin{aligned}
\xi_{\tilde{A}}(x) & =\sup _{\alpha \in[0,1]} \alpha \cdot \chi_{\alpha \tilde{A}}^{\star}(x)=\max _{\alpha \in[0,1]} \alpha \cdot \chi_{\alpha}^{\star}(x)=\max _{\alpha \in[0,1]} \alpha \cdot\left[1-\chi_{\alpha}(x)\right] \\
& =\sup _{\alpha \in \mathbb{Q}(0,1]} \alpha \cdot \chi_{\alpha \tilde{A}}^{\star}(x)=\max _{\alpha \in \mathbb{Q}(0,1]} \alpha \cdot \chi_{\alpha}^{\star} \tilde{A}(x)=\max _{\alpha \in \mathbb{Q}(0,1]} \alpha \cdot\left[1-\chi_{\alpha} \tilde{A}(x)\right] .
\end{aligned}
$$

Proof. From Remark 4, it follows that $I_{\tilde{A}}^{\circ}=[0,1]$. Using Theorem 1, we can obtain the equalities (9). To prove the equalities (10), let $\xi_{\tilde{A}}(x)=\alpha_{0}$. We first assume that $\alpha_{0}=0$. From the proof of Theorem 1 , we have $x \in{ }_{\alpha} \tilde{A}$ for all $\alpha \in I_{\tilde{A}}^{\circ}=[0,1]$, which says that $\alpha \cdot \chi_{\alpha}^{\star} \tilde{A}(x)=0$ for all $\alpha \in \mathbb{Q}(0,1]$. It follows that

$$
\xi_{\tilde{A}}(x)=\alpha_{0}=0=\sup _{\alpha \in \mathbb{Q}(0,1]} \alpha \cdot \chi_{\alpha}^{\star} \tilde{A}(x) .
$$

Now we assume that $0<\alpha_{0}$. Using (9), we have

$$
0<\xi_{\tilde{A}}(x)=\alpha_{0}=\sup _{\alpha \in[0,1]} \alpha \cdot \chi_{\alpha}^{\star} \tilde{A}(x) \geq \sup _{\alpha \in \mathbb{Q}(0,1]} \alpha \cdot \chi_{\alpha \tilde{A}}^{\star}(x) .
$$

Since $\alpha_{0}>0$, from the proof of Theorem 1 we have $x \notin{ }_{\alpha} \tilde{A}$ for $\alpha \in I_{\tilde{A}}^{\circ}=[0,1]$ with $\alpha<\alpha_{0}$. The denseness also says that there exists a sequence $\left\{\alpha_{n}\right\}_{n=1}^{\infty}$ in $\mathbb{Q}(0,1]$ such that $\alpha_{n} \uparrow \alpha_{0}$ with $\alpha_{n}<\alpha_{0}$. It follows that $x \notin \alpha_{n} \tilde{A}$ for all $n$. Let $\Gamma=\left\{\alpha_{n}\right\}_{n=1}^{\infty} \subset \mathbb{Q}(0,1]$. Then we have

$$
\xi_{\tilde{A}}(x)=\alpha_{0}=\lim _{n} \alpha_{n}=\sup _{n} \alpha_{n}=\sup _{n} \alpha_{n} \cdot \chi_{\alpha_{n} \tilde{A}}^{\star}(x)=\sup _{\alpha \in \Gamma} \alpha \cdot \chi_{\alpha}^{\star} \tilde{A}(x) \leq \sup _{\alpha \in \mathbb{Q}(0,1]} \alpha \cdot \chi_{\alpha}^{\star} \tilde{A}(x) \text {. }
$$

Combining (11) and (12), we obtain the equality

$$
\xi_{\tilde{A}}(x)=\sup _{\alpha \in \mathbb{Q}(0,1]} \alpha \cdot \chi_{\alpha}^{\star} \tilde{A}(x) .
$$

This completes the proof.

\section{Dual Arithmetics of Fuzzy Sets}

Let $\tilde{A}$ and $\tilde{B}$ be fuzzy numbers in $\mathbb{R}$; that is, $\tilde{A}$ and $\tilde{B}$ are normal fuzzy sets in $\mathbb{R}$ satisfying some elegant structures such that their $\alpha$-level sets turn into the bounded closed intervals in $\mathbb{R}$. Then we have the following well-known equality

$$
(\tilde{A} \odot \tilde{B})_{\alpha}=\tilde{A}_{\alpha} \circ \tilde{B}_{\alpha} \equiv\left\{a \circ b: a \in \tilde{A}_{\alpha} \text { and } b \in \tilde{B}_{\alpha}\right\} \text { for } \alpha \in[0,1],
$$

where the upper $\alpha$-level sets are considered. For convenience, we use the same notation $\circ$ to denote the operations for the $\alpha$-level sets $\tilde{A}_{\alpha} \circ \tilde{B}_{\alpha}$ and the real numbers $a \circ b$. In this paper, we shall consider 
the general fuzzy sets in $\mathbb{R}$ rather than the fuzzy numbers to establish the similar equality based on the lower $\alpha$-level sets.

Let $\odot$ denote any one of the four basic arithmetic operations $\oplus, \ominus, \otimes, \oslash$ between fuzzy sets $\tilde{A}$ and $\tilde{B}$ in $\mathbb{R}$. The membership function of $\tilde{A} \odot \tilde{B}$ is defined by

$$
\xi_{\tilde{A} \odot \tilde{B}}(z)=\sup _{\{(x, y) \in U \times U: z=x \circ y\}} \min \left\{\tilde{\zeta}_{\tilde{A}}(x), \xi_{\tilde{B}}(y)\right\}
$$

for all $z \in \mathbb{R}$, where the operation $\circ \in\{+,-, \times, /\}$, respectively. Since the 0-level sets $\tilde{A}_{0}$ and $\tilde{B}_{0}$ are the proper domain of $\tilde{A}$ and $\tilde{B}$, respectively, i.e., $\xi_{\tilde{A}}(x)=0$ for $x \notin \tilde{A}_{0}$ and $\xi_{\tilde{B}}(x)=0$ for $x \notin \tilde{B}_{0}$, we have

$$
\xi_{\tilde{A} \odot \tilde{B}}(z)=\sup _{\{(x, y) \in U \times U: z=x \circ y\}} \min \left\{\xi_{\tilde{A}}(x), \xi_{\tilde{B}}(y)\right\}=\sup _{\left\{(x, y) \in \tilde{A}_{0} \times \tilde{B}_{0}: z=x \circ y\right\}} \min \left\{\xi_{\tilde{A}}(x), \xi_{\tilde{B}}(y)\right\} .
$$

Inspired by the above expression (13), we define a new operation between $\tilde{A}$ and $\tilde{B}$ using the dual membership functions as follows

$$
\xi_{\tilde{A} \boxminus \tilde{B}}(z)=\inf _{\left\{(x, y) \in \tilde{A}_{0} \times \tilde{B}_{0}: z=x \circ y\right\}} \max \left\{\xi_{\tilde{A}^{\star}}(x), \xi_{\tilde{B}^{\star}}(y)\right\}=\inf _{\left\{(x, y) \in \tilde{A}_{0} \times \tilde{B}_{0}: z=x \circ y\right\}} \max \left\{\xi_{\tilde{A}^{c}}(x), \xi_{\tilde{B}^{c}}(y)\right\} .
$$

We need to emphasize that

$$
\inf _{\left\{(x, y) \in \tilde{A}_{0} \times \tilde{B}_{0}: z=x \circ y\right\}} \max \left\{\xi_{\tilde{A}^{\star}}(x), \xi_{\tilde{B}^{\star}}(y)\right\} \neq \inf _{\{(x, y): z=x \circ y\}} \max \left\{\tilde{\zeta}_{\tilde{A}^{\star}}(x), \xi_{\tilde{B}^{\star}}(y)\right\} .
$$

However, this operation $\tilde{A} \boxminus \tilde{B}$ is reasonable, since we consider the proper domains as shown in (13). Then we have

$$
\begin{aligned}
\xi_{\tilde{A} \boxminus \tilde{B}}(z) & =\inf _{\left\{(x, y) \in \tilde{A}_{0} \times \tilde{B}_{0}: z=x \circ y\right\}} \max \left\{1-\xi_{\tilde{A}}(x), 1-\xi_{\tilde{B}}(y)\right\} \\
& =1-\sup _{\left\{(x, y) \in \tilde{A}_{0} \times \tilde{B}_{0}: z=x \circ y\right\}} \min \left\{\xi_{\tilde{A}}(x), \xi_{\tilde{B}}(y)\right\} \\
& =1-\xi_{\tilde{A} \odot \tilde{B}}(z),
\end{aligned}
$$

which implies

$$
\xi_{\tilde{A} \odot \tilde{B}}(z)+\xi_{\tilde{A} \boxminus \tilde{B}}(z)=1 .
$$

Therefore we say that $\tilde{A} \boxminus \tilde{B}$ is the dual arithmetic of $\tilde{A} \odot \tilde{B}$. This means that, instead of calculating $\tilde{A} \odot \tilde{B}$, we can alternatively calculate $\tilde{A} \odot \tilde{B}$ and use the duality (14) to recover $\tilde{A} \odot \tilde{B}$. We are going to study the lower $\alpha$-level sets of dual arithmetic $\tilde{A} \odot \tilde{B}$ and establish the relationships between $\tilde{A} \odot \tilde{B}$ and $\tilde{A} \square \tilde{B}$.

Let $\tilde{A}$ and $\tilde{B}$ be two fuzzy sets in $\mathbb{R}$ with membership functions $\xi_{\tilde{A}}$ and $\xi_{\tilde{B}}$, respectively. Let

$$
\alpha_{\tilde{A}}^{*}=\sup \mathcal{R}\left(\xi_{\tilde{A}}\right) \text { and } \alpha_{\tilde{B}}^{*}=\sup \mathcal{R}\left(\xi_{\tilde{B}}\right) .
$$

From Proposition 1 and (3), we see that $\tilde{A}_{\alpha} \neq \varnothing$ for $\alpha \in I_{\tilde{A}^{\prime}}^{*}$ where $I_{\tilde{A}}^{*}$ is given by

$$
I_{\tilde{A}}^{*}= \begin{cases}{\left[0, \alpha_{\tilde{A}}^{*}\right),} & \text { if } \max \mathcal{R}\left(\xi_{\tilde{A}}\right) \text { does not exist } \\ {\left[0, \alpha_{\tilde{A}}^{*}\right],} & \text { if } \max \mathcal{R}\left(\xi_{\tilde{A}}\right) \text { exists. }\end{cases}
$$

Similarly, we also see that $\tilde{B}_{\alpha} \neq \varnothing$ for $\alpha \in I_{\tilde{B}^{\prime}}^{*}$ where $I_{\widetilde{B}}^{*}$ is given by 


$$
I_{\tilde{B}}^{*}= \begin{cases}{\left[0, \alpha_{\tilde{B}}^{*}\right),} & \text { if } \max \mathcal{R}\left(\xi_{\tilde{B}}\right) \text { does not exist } \\ {\left[0, \alpha_{\tilde{B}}^{*}\right],} & \text { if } \max \mathcal{R}\left(\xi_{\tilde{B}}\right) \text { exists. }\end{cases}
$$

For further discussion, we need a simple lemma.

Lemma 1. Let $f$ be a real-valued function defined on $A$, and let $k$ be a constant. Then

$$
\sup _{x \in A} \min \{f(x), k\}=\min \left\{\sup _{x \in A} f(x), k\right\}
$$

and

$$
\inf _{x \in A} \max \{f(x), k\}=\max \left\{\inf _{x \in A} f(x), k\right\}
$$

Proof. We have

$$
\min \left\{\sup _{x \in A} f(x), k\right\}= \begin{cases}k, & \text { if there exists } x \in A \text { such that } f(x)>k \\ \sup _{x \in A} f(x), & \text { if } f(x) \leq k \text { for all } x \in A .\end{cases}
$$

and

$$
\begin{aligned}
& \sup _{x \in A} \min \{f(x), k\}=\left\{\begin{array}{c}
\max \left\{\sup _{\{x \in A: f(x)>k\}} \min \{f(x), k\}, \sup _{\{x \in A: f(x) \leq k\}} \min \{f(x), k\}\right\}, \\
\quad \text { if there exists } x \in A \text { such that } f(x)>k
\end{array}\right. \\
& =\left\{\begin{array}{c}
\max \left\{k, \sup _{\{x \in A: f(x) \leq k\}} f(x)\right\}, \\
\quad \text { if there exists } x \in A \text { such that } f(x)>k \\
\sup _{x \in A} f(x), \quad \text { if } f(x) \leq k \text { for all } x \in A
\end{array} .\right. \\
& = \begin{cases}k, & \text { if there exists } x \in A \text { such that } f(x)>k \\
\sup _{x \in A} f(x), & \text { if } f(x) \leq k \text { for all } x \in A .\end{cases}
\end{aligned}
$$

Another equality can be similarly obtained. This completes the proof.

Proposition 7. Let $\tilde{A}$ and $\tilde{B}$ be two fuzzy sets in $\mathbb{R}$. Then the following statements hold true.

(i) We have

$$
\alpha_{\tilde{A} \odot \tilde{B}}^{*} \equiv \sup \mathcal{R}\left(\xi_{\tilde{A} \odot \tilde{B}}\right)=\min \left\{\sup \mathcal{R}\left(\xi_{\tilde{A}}\right), \sup \mathcal{R}\left(\xi_{\tilde{B}}\right)\right\}
$$

with

$$
I_{\tilde{A} \odot \tilde{B}}^{*}= \begin{cases}{\left[0, \alpha_{\tilde{A} \odot \tilde{B}}^{*}\right),} & \text { if } \max \mathcal{R}\left(\xi_{\tilde{A} \odot \tilde{B}}\right) \text { does not exist } \\ {\left[0, \alpha_{\tilde{A} \odot \tilde{B}}^{*}\right],} & \text { if } \max \mathcal{R}\left(\xi_{\tilde{A} \odot \tilde{B}}\right) \text { exists }\end{cases}
$$

We also have $(\tilde{A} \odot \tilde{B})_{\alpha} \neq \varnothing$ for $\alpha \in I_{\tilde{A} \odot \tilde{B}}^{*}$ and $(\tilde{A} \odot \tilde{B})_{\alpha}=\varnothing$ for $\alpha \notin I_{\tilde{A} \odot \tilde{B}}^{*}$.

(ii) We have

$$
\begin{aligned}
\alpha_{\tilde{A} \sqcup \tilde{B}}^{\circ} \equiv \inf \mathcal{R}\left(\xi_{\tilde{A} \boxminus \tilde{B}}\right) & =\max \left\{\inf \mathcal{R}\left(\xi_{\tilde{A}^{\star}}\right), \inf \mathcal{R}\left(\xi_{\tilde{B}^{\star}}\right)\right\} \\
& =\max \left\{1-\sup \mathcal{R}\left(\xi_{\tilde{A}}\right), 1-\sup \mathcal{R}\left(\xi_{\tilde{B}}\right)\right\}
\end{aligned}
$$


with

$$
I_{\tilde{A} \boxminus \tilde{B}}^{\circ}= \begin{cases}\left(\alpha_{\tilde{A} \boxminus \tilde{B}^{\prime}}^{\circ} 1\right], & \text { if } \min \mathcal{R}\left(\xi_{\tilde{A} \boxminus \tilde{B}}\right) \text { does not exist } \\ {\left[\alpha_{\tilde{A} \boxminus \tilde{B}^{\prime}}^{\circ} 1\right],} & \text { if } \min \mathcal{R}\left(\xi_{\tilde{A} \square \tilde{B}}\right) \text { exists }\end{cases}
$$

We also have $\alpha(\tilde{A} \boxminus \tilde{B}) \neq \varnothing$ for $\alpha \in I_{\tilde{A} \boxminus \tilde{B}}^{\circ}$ and $\alpha(\tilde{A} \square \tilde{B})=\varnothing$ for $\alpha \notin I_{\tilde{A} \square \tilde{B}}^{\circ}$.

Proof. To prove part (i), let $\alpha_{\tilde{A}}^{*}$ and $\alpha_{\tilde{B}}^{*}$ be defined in (15). Then $\xi_{\tilde{A}}(x) \leq \alpha_{\tilde{A}}^{*}$ and $\xi_{\tilde{B}}(y) \leq \alpha_{\tilde{B}}^{*}$ for all $x \in \tilde{A}_{0}$ and $y \in \tilde{B}_{0}$. It follows that

$$
\min \left\{\tilde{\zeta}_{\tilde{A}}(x), \xi_{\tilde{B}}(y)\right\} \leq \min \left\{\alpha_{\tilde{A}^{\prime}}^{*} \alpha_{\tilde{B}}^{*}\right\}
$$

for all $x \in \tilde{A}_{0}$ and $y \in \tilde{B}_{0}$, which implies

$$
\begin{aligned}
\tilde{\zeta}_{\tilde{A} \odot \tilde{B}}(z) & =\sup _{\{(x, y): z=x \circ y\}} \min \left\{\xi_{\tilde{A}}(x), \xi_{\tilde{B}}(y)\right\} \\
& =\sup _{\left\{(x, y) \in \tilde{A}_{0} \times \tilde{B}_{0}: z=x \circ y\right\}} \min \left\{\tilde{\xi}_{\tilde{A}}(x), \xi_{\tilde{B}}(y)\right\} \leq \min \left\{\alpha_{\tilde{A}}^{*}, \alpha_{\tilde{B}}^{*}\right\}
\end{aligned}
$$

for all $z \in U$. This says that $\min \left\{\alpha_{\tilde{A}^{\prime}}^{*} \alpha_{\tilde{B}}^{*}\right\}$ is an upper bound of function $\xi_{\tilde{A} \odot \tilde{B}}$. Suppose that $\eta \geq \xi_{\tilde{A} \odot \tilde{B}}(z)$ for all $z \in U$. Then $\eta \geq \min \left\{\tilde{\xi}_{\tilde{A}}(x), \xi_{\tilde{B}}(y)\right\}$ for all $x \in \tilde{A}_{0}, y \in \tilde{B}_{0}$ and $z \in U$. Using Lemma 1, we have

$$
\eta \geq \sup _{x \in \tilde{A}_{0}} \sup _{y \in \tilde{B}_{0}} \min \left\{\xi_{\tilde{A}}(x), \xi_{\tilde{B}}(y)\right\}=\min \left\{\sup _{x \in \tilde{A}_{0}} \xi_{\tilde{A}}(x), \sup _{y \in \tilde{B}_{0}} \xi_{\tilde{B}}(y)\right\}=\min \left\{\alpha_{\tilde{A}}^{*}, \alpha_{\tilde{B}}^{*}\right\} .
$$

This says that $\min \left\{\alpha_{\tilde{A}}^{*}, \alpha_{\tilde{B}}^{*}\right\}$ is a least upper bound of function $\xi_{\tilde{A} \odot \tilde{B}}$. By the definition of supremum, we obtain the desired equality (16). The interval $I_{\tilde{A} \odot \tilde{B}}^{*}$ follows from Proposition 1 immediately.

To prove part (ii), we first note that

$$
\inf _{x \in \tilde{A}_{0}} \xi_{\tilde{A}^{\star}}(x)=1-\sup _{x \in \tilde{A}_{0}} \xi_{\tilde{A}}(x)=1-\alpha_{\tilde{A}}^{*} \text { and } \inf _{x \in \tilde{A}_{0}} \xi_{\tilde{B}^{\star}}(x)=1-\sup _{x \in \tilde{A}_{0}} \xi_{\tilde{B}}(x)=1-\alpha_{\tilde{B}}^{*} .
$$

Since

$$
\xi_{\tilde{A}^{\star}}(x) \geq \inf _{x \in \tilde{A}_{0}} \xi_{\tilde{A}^{\star}}(x)=1-\alpha_{\tilde{A}^{\prime}}^{*} \text { and } \xi_{\tilde{B}^{\star}}(x) \geq \inf _{x \in \tilde{A}_{0}} \xi_{\tilde{B}^{\star}}(x)=1-\alpha_{\tilde{B}}^{*}
$$

for all $x \in \tilde{A}_{0}$ and $y \in \tilde{B}_{0}$, it follows that

$$
\max \left\{\xi_{\tilde{A}^{\star}}(x), \xi_{\tilde{B}^{\star}}(y)\right\} \geq \max \left\{1-\alpha_{\tilde{A}^{\prime}}^{*} 1-\alpha_{\tilde{B}}^{*}\right\}
$$

for all $x \in \tilde{A}_{0}$ and $y \in \tilde{B}_{0}$, which implies

$$
\xi_{\tilde{A} \sqcup \tilde{B}}(z)=\inf _{\left\{(x, y) \in \tilde{A}_{0} \times \tilde{B}_{0}: z=x * y\right\}} \max \left\{\xi_{\tilde{A}^{\star}}(x), \xi_{\tilde{B}^{\star}}(y)\right\} \geq \max \left\{1-\alpha_{\tilde{A}^{\prime}}^{*} 1-\alpha_{\tilde{B}}^{*}\right\}
$$

for all $z \in U$. This says that $\max \left\{1-\alpha_{\tilde{A}^{\prime}}^{*}, 1-\alpha_{\tilde{B}}^{*}\right\}$ is a lower bound of function $\xi_{\tilde{A}} \cdot \tilde{B}$. Suppose that $\zeta \leq \xi_{\tilde{A} \boxminus \tilde{B}}(z)$ for all $z \in U$. Then $\zeta \leq \max \left\{\tilde{\zeta}_{\tilde{A}^{\star}}(x), \tilde{\zeta}_{\tilde{B}^{\star}}(y)\right\}$ for all $x \in \tilde{A}_{0}, y \in \tilde{B}_{0}$ and $z \in U$. Using Lemma 1, we have

$$
\zeta \leq \inf _{x \in \tilde{A}_{0}} \inf _{y \in \tilde{B}_{0}} \max \left\{\tilde{\zeta}_{\tilde{A}^{\star}}(x), \xi_{\tilde{B}^{\star}}(y)\right\}=\max \left\{\inf _{x \in \tilde{A}_{0}} \xi_{\tilde{A}^{\star}}(x), \inf _{y \in \tilde{B}_{0}} \xi_{\tilde{B}^{\star}}(y)\right\}=\max \left\{1-\alpha_{\tilde{A}^{\prime}}^{*} 1-\alpha_{\tilde{B}}^{*}\right\} .
$$


This says that $\max \left\{1-\alpha_{\tilde{A}^{\prime}}^{*} 1-\alpha_{\tilde{B}}^{*}\right\}$ is a greatest lower bound of function $\tilde{\zeta}_{\tilde{A}}^{*} \boxminus \tilde{B}$. By the definition of infimum, we obtain the desired equality (17). The interval $I_{\tilde{A} \boxminus \tilde{B}}^{\circ}$ follows from Proposition 2 immediately. This completes the proof.

We write $I^{*, \cap}=I_{\tilde{A}}^{*} \cap I_{\tilde{B}}^{*}$. Then $I^{*, \cap} \neq \varnothing$ is given by

$$
I^{*, \cap}= \begin{cases}{\left[0, \alpha_{\tilde{A}}^{*}\right) \cap\left[0, \alpha_{\tilde{B}}^{*}\right),} & \text { if } \max \mathcal{R}\left(\xi_{\tilde{A}}\right) \text { and } \max \mathcal{R}\left(\xi_{\tilde{B}}\right) \text { does not exist } \\ {\left[0, \alpha_{\tilde{A}}^{*}\right] \cap\left[0, \alpha_{\tilde{B}}^{*}\right),} & \text { if } \max \mathcal{R}\left(\xi_{\tilde{A}}\right) \text { exists and } \max \mathcal{R}\left(\xi_{\tilde{B}}\right) \text { does not exist } \\ {\left[0, \alpha_{\tilde{A}}^{*}\right) \cap\left[0, \alpha_{\tilde{B}}^{*}\right],} & \text { if } \max \mathcal{R}\left(\xi_{\tilde{A}}\right) \text { does not exist and } \max \mathcal{R}\left(\xi_{\tilde{B}}\right) \text { exists } \\ {\left[0, \alpha_{\tilde{A}}^{*}\right] \cap\left[0, \alpha_{\tilde{B}}^{*}\right],} & \text { if } \max \mathcal{R}\left(\xi_{\tilde{A}}\right) \text { and } \max \mathcal{R}\left(\xi_{\tilde{B}}\right) \text { exist. }\end{cases}
$$

From part (i) of Proposition 7 by referring to (16), we see that

$$
I^{*, \cap}=I_{\tilde{A} \odot \tilde{B}}^{*}
$$

Let $S$ be a nonempty subset in a topological space $(U, \tau)$. Recall that $S$ is compact if and only if, for every sequence $\left\{x_{n}\right\}_{n=1}^{\infty}$ in $S$, there exists a convergent subsequence $\left\{x_{n_{k}}\right\}_{k=1}^{\infty}$ in $S$. If the limit of $\left\{x_{n_{k}}\right\}_{k=1}^{\infty}$ is denoted by $x_{0}$, then $x_{0}$ is in $S$. In particular, if $U=\mathbb{R}^{n}$, then $S$ is compact if and only if $S$ is closed and bounded. We need a useful lemma.

Lemma 2. (Royden ([18] p. 161)). Let $U$ be a topological space, and let $K$ be a compact subset of $U$. Let $f$ be a real-valued function defined on $U$.

(i) If $f$ is lower semi-continuous, then $f$ assumes its minimum on a compact subset of $U$; that is, the infimum is attained in the following sense

$$
\inf _{x \in K} f(x)=\min _{x \in K} f(x) .
$$

(ii) If $f$ is upper semi-continuous, then $f$ assumes its maximum on a compact subset of $U$; that is, the supremum is attained in the following sense

$$
\sup _{x \in K} f(x)=\max _{x \in K} f(x) .
$$

If $\alpha \notin I^{*, \cap}$ then $\tilde{A}_{\alpha}=\varnothing$ or $\tilde{B}_{\alpha}=\varnothing$. Therefore, in order to consider the operation

$$
\tilde{A}_{\alpha} \circ \tilde{B}_{\alpha} \equiv\left\{a \circ b: a \in \tilde{A}_{\alpha} \text { and } b \in \tilde{B}_{\alpha}\right\},
$$

we need to take $\alpha \in I^{*, \cap}$. We also remark that if $\tilde{A}$ and $\tilde{B}$ are normal fuzzy sets then $I^{*, \cap}=[0,1]$.

Theorem 3. Let $\tilde{A}$ and $\tilde{B}$ be two fuzzy sets in $\mathbb{R}$ with the dual fuzzy sets $\tilde{A}^{\star}$ and $\tilde{B}^{\star}$, respectively. Suppose that the arithmetic operations $\odot \in\{\oplus, \ominus, \otimes\}$ correspond to the operations $\circ \in\{+,-, *\}$. Then the following statements hold true.

(i) We have $(\tilde{A} \odot \tilde{B})_{\alpha}=\varnothing$ for $\alpha \notin I^{*, \cap}$.

(ii) We have the following inclusion

$$
(\tilde{A} \odot \tilde{B})_{\alpha} \supseteq \tilde{A}_{\alpha} \circ \tilde{B}_{\alpha}={ }_{1-\alpha} \tilde{A}^{\star} \circ{ }_{1-\alpha} \tilde{B}^{\star} \text { for all } \alpha \in I^{*, \cap} .
$$

(iii) Suppose that the membership functions of $\tilde{A}$ and $\tilde{B}$ are upper semi-continuous. Then

$$
(\tilde{A} \odot \tilde{B})_{\alpha}=\tilde{A}_{\alpha} \circ \tilde{B}_{\alpha} \text { for all } \alpha \in I^{*, \cap} \text { with } \alpha>0
$$


and

$$
(\tilde{A} \odot \tilde{B})_{0}=\operatorname{cl}(\tilde{A} \odot \tilde{B})_{0+}=\operatorname{cl}\left(\bigcup_{0<\alpha \in I^{*, \cap}}\left(\tilde{A}_{\alpha} \circ \tilde{B}_{\alpha}\right)\right) .
$$

(iv) Suppose that the membership functions of $\tilde{A}$ and $\tilde{B}$ are upper semi-continuous, and that the supports $\tilde{A}_{0+}$ and $\tilde{B}_{0+}$ are bounded. Then

$$
(\tilde{A} \odot \tilde{B})_{\alpha}=\tilde{A}_{\alpha} \circ \tilde{B}_{\alpha}={ }_{1-\alpha} \tilde{A}^{\star} \circ_{1-\alpha} \tilde{B}^{\star} \text { for all } \alpha \in I^{*, \cap} .
$$

Proof. To prove part (i), since $I^{*, \cap}=I_{\tilde{A} \odot \tilde{B}}^{*}$ by (19), Proposition 1 says that $(\tilde{A} \odot \tilde{B})_{\alpha}=\varnothing$ for $\alpha \notin I_{\tilde{A} \odot \tilde{B}}^{*}=I^{*, \cap}$.

To prove part (ii), for $\alpha \in I^{*, \cap}$ with $\alpha>0$ and $z_{\alpha} \in \tilde{A}_{\alpha} \circ \tilde{B}_{\alpha}$, since $\tilde{A}_{\alpha} \neq \varnothing$ and $\tilde{B}_{\alpha} \neq \varnothing$, there exist $x_{\alpha} \in \tilde{A}_{\alpha}$ and $y_{\alpha} \in \tilde{B}_{\alpha}$ such that $z_{\alpha}=x_{\alpha} \circ y_{\alpha}$ for $\circ \in\{+,-, *\}$, where $\xi_{\tilde{A}}\left(x_{\alpha}\right) \geq \alpha$ and $\xi_{\tilde{B}}\left(y_{\alpha}\right) \geq \alpha$. Therefore, we have

$$
\xi_{\tilde{A} \odot \tilde{B}}\left(z_{\alpha}\right)=\sup _{\left\{(x, y): z_{\alpha}=x \circ y\right\}} \min \left\{\xi_{\tilde{A}}(x), \xi_{\tilde{B}}(y)\right\} \geq \min \left\{\xi_{\tilde{A}}\left(x_{\alpha}\right), \xi_{\tilde{B}}\left(y_{\alpha}\right)\right\} \geq \alpha,
$$

which says that $z_{\alpha} \in(\tilde{A} \odot \tilde{B})_{\alpha}$. This shows that $\tilde{A}_{\alpha} \circ \tilde{B}_{\alpha} \subseteq(\tilde{A} \odot \tilde{B})_{\alpha}$ for $\alpha \in I^{*, \cap}$ with $\alpha>0$.

Now, for $\alpha=0$ and $z_{0} \in \tilde{A}_{0} \circ \tilde{B}_{0}$, there also exist $x_{0} \in \tilde{A}_{0}$ and $y_{0} \in \tilde{B}_{0}$ such that $z_{0}=x_{0} \circ y_{0}$ for $\circ \in\{+,-, *\}$. Since

$$
\tilde{A}_{0}=\operatorname{cl}\left(\left\{x \in \mathbb{R}: \xi_{\tilde{A}}(x)>0\right\}\right) \text { and } \tilde{B}_{0}=\operatorname{cl}\left(\left\{y \in \mathbb{R}: \xi_{\tilde{B}}(y)>0\right\}\right),
$$

there exist sequence $\left\{x_{n}\right\}_{n=1}^{\infty}$ in $\left\{x \in \mathbb{R}: \xi_{\tilde{A}}(x)>0\right\}$ and sequence $\left\{y_{n}\right\}_{n=1}^{\infty}$ in $\left\{y \in \mathbb{R}: \xi_{\tilde{B}}(y)>0\right\}$ such that $x_{n} \rightarrow x_{0}$ and $y_{n} \rightarrow y_{0}$ as $n \rightarrow \infty$. Let $z_{n}=x_{n} \circ y_{n}$. Then we see that $z_{n} \rightarrow x_{0} \circ y_{0}=z_{0}$, since the binary operation $\circ \in\{+,-, *\}$ is continuous. We also have

$$
\xi_{\tilde{A} \odot \tilde{B}}\left(z_{n}\right)=\sup _{\left\{(x, y): z_{n}=x \circ y\right\}} \min \left\{\xi_{\tilde{A}}(x), \xi_{\tilde{B}}(y)\right\} \geq \min \left\{\xi_{\tilde{A}}\left(x_{n}\right), \xi_{\tilde{B}}\left(y_{n}\right)\right\}>0,
$$

which says that $z_{n} \in\left\{z \in \mathbb{R}: \xi_{\tilde{A} \odot \tilde{B}}(z)>0\right\}$. Since $z_{n} \rightarrow z_{0}$, it means that

$$
z_{0} \in \operatorname{cl}\left(\left\{z \in \mathbb{R}: \xi_{\tilde{A} \odot \tilde{B}}(z)>0\right\}\right)=(\tilde{A} \odot \tilde{B})_{0} .
$$

This shows that $\tilde{A}_{0} \circ \tilde{B}_{0} \subseteq(\tilde{A} \odot \tilde{B})_{0}$. Therefore we conclude that $\tilde{A}_{\alpha} \circ \tilde{B}_{\alpha} \subseteq(\tilde{A} \odot \tilde{B})_{\alpha}$ for $\alpha \in I^{*, \cap}$.

To prove part (iii), in order to prove another direction of inclusion, we further assume that the membership functions of $\tilde{A}$ and $\tilde{B}$ are upper semi-continuous; that is, the nonempty $\alpha$-level sets $\tilde{A}_{\alpha}$ and $\tilde{B}_{\alpha}$ are closed subsets of $\mathbb{R}$ for all $\alpha \in I^{*, \cap}$. Given any $\alpha \in I^{*, \cap}$ with $\alpha>0$ and $z_{\alpha} \in(\tilde{A} \odot \tilde{B})_{\alpha}$, we have

$$
\sup _{\left\{(x, y): z_{\alpha}=x \circ y\right\}} \min \left\{\tilde{\xi}_{\tilde{A}}(x), \xi_{\tilde{B}}(y)\right\}=\xi_{\tilde{A} \odot \tilde{B}}\left(z_{\alpha}\right) \geq \alpha .
$$

Since $z_{\alpha}$ is finite, it is clear to see that $F \equiv\left\{(x, y): z_{\alpha}=x \circ y\right\}$ is a bounded subset of $\mathbb{R}^{2}$. We also see that the function $g(x, y)=x \circ y$ is continuous on $\mathbb{R}^{2}$. Since the singleton set $\left\{z_{\alpha}\right\}$ is a closed subset of $\mathbb{R}$, it follows that the inverse image $F=g^{-1}\left(\left\{z_{\alpha}\right\}\right)$ of $\left\{z_{\alpha}\right\}$ is also a closed subset of $\mathbb{R}^{2}$. This says that $F$ is a compact subset of $\mathbb{R}^{2}$. Now we want to show that the function $f(x, y)=\min \left\{\tilde{\zeta}_{\tilde{A}}(x), \tilde{\zeta}_{\tilde{B}}(y)\right\}$ is upper semi-continuous, i.e., we want to show that $\{(x, y): f(x, y) \geq \alpha\}$ is a closed subset of $\mathbb{R}^{2}$ for any $\alpha \in \mathbb{R}$.

- $\quad$ For $\alpha \in I^{*, \cap}$ with $\alpha>0$, i.e., $\tilde{A}_{\alpha} \neq \varnothing$ and $\tilde{B}_{\alpha} \neq \varnothing$, we have

$$
\begin{aligned}
\{(x, y): f(x, y) \geq \alpha\} & =\left\{(x, y): \xi_{\tilde{A}}(x) \geq \alpha \text { and } \xi_{\tilde{B}}(y) \geq \alpha\right\} \\
& =\left\{(x, y): x \in \tilde{A}_{\alpha} \text { and } y \in \tilde{B}_{\alpha}\right\}=\tilde{A}_{\alpha} \times \tilde{B}_{\alpha}
\end{aligned}
$$


which is a closed subset of $\mathbb{R}^{2}$, since $\tilde{A}_{\alpha}$ and $\tilde{B}_{\alpha}$ are closed subsets of $\mathbb{R}$.

- If $\alpha \leq 0$, then $\{(x, y): f(x, y) \geq \alpha\}=\mathbb{R}^{2}$ is a closed subset of $\mathbb{R}^{2}$.

- If $\alpha \notin I^{*, \cap}$ with $\alpha>0$ then $\tilde{A}_{\alpha}=\varnothing$ or $\tilde{B}_{\alpha}=\varnothing$. Suppose that $\tilde{A}_{\alpha}=\varnothing$. Then $\xi_{\tilde{A}}(x)<\alpha$ for all $x \in \mathbb{R}$. Similarly, if $\tilde{B}_{\alpha}=\varnothing$, then $\xi_{\tilde{B}}(y)<\alpha$ for all $y \in \mathbb{R}$. Therefore we conclude that $f(x, y)<\alpha$ for all $(x, y) \in \mathbb{R}^{2}$, which implies $\{(x, y): f(x, y) \geq \alpha\}=\varnothing$ that is also a closed subset of $\mathbb{R}^{2}$.

Therefore the function $f(x, y)$ is indeed upper semi-continuous. By Lemma 2, the function $f$ assumes maximum on $F$; that is, from (20), we have

$$
\max _{(x, y) \in F} f(x, y)=\max _{\left\{(x, y): z_{\alpha}=x \circ y\right\}} f(x, y)=\sup _{\left\{(x, y): z_{\alpha}=x \circ y\right\}} f(x, y) \geq \alpha .
$$

In other words, there exists $\left(x_{\alpha}, y_{\alpha}\right) \in F$ such that $z_{\alpha}=x_{\alpha} \circ y_{\alpha}$ and

$$
\min \left\{\xi_{\tilde{A}}\left(x_{\alpha}\right), \xi_{\tilde{B}}\left(y_{\alpha}\right)\right\}=f\left(x_{\alpha}, y_{\alpha}\right)=\max _{(x, y) \in F} f(x, y) \geq \alpha,
$$

i.e., $\xi_{\tilde{A}}\left(x_{\alpha}\right) \geq \alpha$ and $\xi_{\tilde{B}}\left(y_{\alpha}\right) \geq \alpha$. Therefore, we obtain $x_{\alpha} \in \tilde{A}_{\alpha}$ and $y_{\alpha} \in \tilde{B}_{\alpha}$, which says that $z_{\alpha} \in \tilde{A}_{\alpha} \circ \tilde{B}_{\alpha}$, i.e., $(\tilde{A} \odot \tilde{B})_{\alpha} \subseteq \tilde{A}_{\alpha} \circ \tilde{B}_{\alpha}$ for all $\alpha \in I^{*, \cap}$ with $\alpha>0$. Using part (i), we obtain the desired equality. We also have

$$
\begin{aligned}
(\tilde{A} \odot \tilde{B})_{0} & =\operatorname{cl}(\tilde{A} \odot \tilde{B})_{0+}=\mathrm{cl}\left(\bigcup_{0<\alpha \leq 1}(\tilde{A} \odot \tilde{B})_{\alpha}\right) \text { (using part (iv) of Proposition 4) } \\
& =\operatorname{cl}\left(\bigcup_{0<\alpha \in I^{*, \cap}}\left(\tilde{A}_{\alpha} \circ \tilde{B}_{\alpha}\right)\right) \text { (using part (i)) } \\
& =\operatorname{cl}\left(\bigcup_{0<\alpha \in I^{*}, \cap}\left(\tilde{A}_{\alpha} \circ \tilde{B}_{\alpha}\right)\right) .
\end{aligned}
$$

To prove part (iv), for $\alpha=0$ and

$$
z_{0} \in(\tilde{A} \odot \tilde{B})_{0}=\operatorname{cl}\left((\tilde{A} \odot \tilde{B})_{0+}\right)=\operatorname{cl}\left(\left\{z \in \mathbb{R}: \tilde{\zeta}_{\tilde{A} \odot \tilde{B}}(z)>0\right\}\right),
$$

there exists a sequence $\left\{z_{n}\right\}_{n=1}^{\infty}$ in the set $\left\{z \in \mathbb{R}: \xi_{\tilde{A} \odot \tilde{B}}(z)>0\right\}$ such that $z_{n} \rightarrow z_{0}$ as $n \rightarrow \infty$. Using the above same arguments by referring to (21), we also have

$$
0<\xi_{\tilde{A} \odot \tilde{B}}\left(z_{n}\right)=\sup _{\left\{(x, y): z_{n}=x \circ y\right\}} \min \left\{\xi_{\tilde{A}}(x), \xi_{\tilde{B}}(y)\right\}=\max _{\left\{(x, y): z_{n}=x \circ y\right\}} \min \left\{\xi_{\tilde{A}}(x), \xi_{\tilde{B}}(y)\right\} .
$$

Therefore, there exist $x_{n}$ and $y_{n}$ such that $z_{n}=x_{n} \circ y_{n}$ and

$$
\min \left\{\xi_{\tilde{A}}\left(x_{n}\right), \xi_{\tilde{B}}\left(y_{n}\right)\right\}=\max _{\left\{(x, y): z_{n}=x \circ y\right\}} \min \left\{\xi_{\tilde{A}}(x), \xi_{\tilde{B}}(y)\right\}>0,
$$

i.e., $\xi_{\tilde{A}}\left(x_{n}\right)>0$ and $\xi_{\tilde{B}}\left(y_{n}\right)>0$. This shows that the sequences $\left\{x_{n}\right\}_{n=1}^{\infty}$ and $\left\{y_{n}\right\}_{n=1}^{\infty}$ are in the supports $\tilde{A}_{0+}$ and $\tilde{B}_{0+}$, respectively. Since $\tilde{A}_{0+}$ and $\tilde{B}_{0+}$ are bounded, i.e., $\left\{x_{n}\right\}_{n=1}^{\infty}$ and $\left\{y_{n}\right\}_{n=1}^{\infty}$ are bounded sequences, there exist convergent subsequences $\left\{x_{n_{k}}\right\}_{k=1}^{\infty}$ and $\left\{y_{n_{k}}\right\}_{k=1}^{\infty}$ of $\left\{x_{n}\right\}_{n=1}^{\infty}$ and $\left\{y_{n}\right\}_{n=1}^{\infty}$, respectively. In other words, we have $x_{n_{k}} \rightarrow x_{0}$ and $y_{n_{k}} \rightarrow y_{0}$ as $k \rightarrow \infty$, where $x_{0} \in$ $\operatorname{cl}\left(\tilde{A}_{0+}\right)=\tilde{A}_{0}$ and $y_{0} \in \operatorname{cl}\left(\tilde{B}_{0+}\right)=\tilde{B}_{0}$. Let $z_{n_{k}}=x_{n_{k}} \circ y_{n_{k}}$. Then $\left\{z_{n_{k}}\right\}_{k=1}^{\infty}$ is a subsequence of $\left\{z_{n}\right\}_{n=1}^{\infty}$, i.e., $z_{n_{k}} \rightarrow z_{0}$ as $k \rightarrow \infty$. Since

$$
z_{0}=\lim _{k \rightarrow \infty} z_{n_{k}}=\lim _{k \rightarrow \infty}\left(x_{n_{k}} \circ y_{n_{k}}\right)=\left(\lim _{k \rightarrow \infty} x_{n_{k}}\right) \circ\left(\lim _{k \rightarrow \infty} y_{n_{k}}\right)=x_{0} \circ y_{0}
$$


which shows that $z_{0} \in \tilde{A}_{0} \circ \tilde{B}_{0}$. Therefore we obtain the inclusion $(\tilde{A} \odot \tilde{B})_{0} \subseteq \tilde{A}_{0} \circ \tilde{B}_{0}$. Using parts (i) and (ii), we obtain the desired equalities. This completes the proof.

We do not consider the operation $\oslash$ in Theorem 3. The reasons is that the case of zero denominator should be avoided. We also remark that the arguments in the proof of Theorem 3 are still available for the operation $\oslash$ by carefully excluding the zero denominator. In order not to complicate the proof of Theorem 3, we omit the case of operation $\oslash$.

Let $\tilde{A}$ and $\tilde{B}$ be two fuzzy sets in $\mathbb{R}$ with the dual fuzzy sets $\tilde{A}^{\star}$ and $\tilde{B}^{\star}$, respectively. We define

$$
\alpha_{\tilde{A}^{\star}}^{\circ}=\inf \mathcal{R}\left(\xi_{\tilde{A}^{\star}}\right) \text { and } \alpha_{\tilde{B}^{\star}}^{\circ}=\inf \mathcal{R}\left(\xi_{\tilde{B}^{\star}}\right) .
$$

From Proposition 2 and (5), we see that ${ }_{\alpha} \tilde{A}^{\star} \neq \varnothing$ for $\alpha \in I_{\tilde{A}^{\star}}^{\circ}$ where $I_{\tilde{A}^{\star}}^{\circ} \neq \varnothing$ is given by

$$
I_{\tilde{A}^{\star}}^{\circ}= \begin{cases}\left(\alpha_{\tilde{A}^{\star}}^{\circ}, 1\right], & \text { if } \min \mathcal{R}\left(\xi_{\tilde{A}^{\star}}\right) \text { does not exist } \\ {\left[\alpha_{\tilde{A}^{\star}}^{\circ} 1\right],} & \text { if } \min \mathcal{R}\left(\xi_{\tilde{A}^{\star}}\right) \text { exists. }\end{cases}
$$

Similarly, we also see that ${ }_{\alpha} \tilde{B}^{\star} \neq \varnothing$ for $\alpha \in I_{\tilde{B}^{\star}}^{\circ}$, where $I_{\tilde{B}^{\star}}^{\circ}$ is given by

$$
I_{\tilde{B}^{\star}}^{\circ}= \begin{cases}\left(\alpha_{\tilde{B}^{\star}}^{\circ}, 1\right], & \text { if } \min \mathcal{R}\left(\xi_{\tilde{B}^{\star}}\right) \text { does not exist } \\ {\left[\alpha_{\tilde{B}^{\star}}^{\circ} 1\right],} & \text { if } \min \mathcal{R}\left(\xi_{\tilde{B}^{\star}}\right) \text { exists. }\end{cases}
$$

We write $I^{\circ, \cap}=I_{\tilde{A}^{\star}}^{\circ} \cap I_{\tilde{B}^{\star}}^{\circ}$. Then $I^{\circ, \cap}$ is given by

$$
I^{\circ, \cap}= \begin{cases}\left(\alpha_{\tilde{A}^{\star}}^{\circ}, 1\right] \cap\left(\alpha_{\tilde{B}^{\star}}^{\circ}, 1\right], & \text { if } \min \mathcal{R}\left(\xi_{\tilde{A}^{\star}}\right) \text { and } \min \mathcal{R}\left(\xi_{\tilde{B}^{\star}}\right) \text { does not exist } \\ {\left[\alpha_{\tilde{A}^{\star}}^{\circ}, 1\right] \cap\left(\alpha_{\tilde{B}^{\star}}^{\circ}, 1\right],} & \text { if } \min \mathcal{R}\left(\xi_{\tilde{A}^{\star}}\right) \text { exists and } \min \mathcal{R}\left(\xi_{\tilde{B}^{\star}}\right) \text { does not exist } \\ \left(\alpha_{\tilde{A}^{\star}}^{\circ}, 1\right] \cap\left[\alpha_{\tilde{B}^{\star}}^{\circ}, 1\right], & \text { if } \min \mathcal{R}\left(\xi_{\tilde{A}^{\star}}\right) \text { does not exist and } \min \mathcal{R}\left(\xi_{\tilde{B}^{\star}}\right) \text { exists } \\ {\left[\alpha_{\tilde{A}^{\star}}^{\circ}, 1\right] \cap\left[\alpha_{\tilde{B}^{\star}}^{\circ}, 1\right],} & \text { if } \min \mathcal{R}\left(\xi_{\tilde{A}^{\star}}\right) \text { and } \min \mathcal{R}\left(\xi_{\tilde{B}^{\star}}\right) \text { exist. }\end{cases}
$$

From part (ii) of Proposition 7 by referring to (17), we see that

$$
I^{\circ, \cap}=I_{\tilde{A} \boxminus \tilde{B}}^{\circ} .
$$

Let $\left(U, \tau_{U}\right)$ be a topological space, and let $A$ be a subset of $U$. Then the subset $A$ can be endowed with a topology $\tau_{A}$ such that $\left(A, \tau_{A}\right)$ is a topological subspace of $\left(U, \tau_{U}\right)$. In other words, the subset $C$ of $A$ is a $\tau_{A}$-closed subset of $A$ if and only if $C=A \cap D$ for some $\tau_{U}$-closed subset $D$ of $U$. In this case, we say that $f:\left(A, \tau_{A}\right) \rightarrow \mathbb{R}$ is upper semi-continuous on $A$ if and only if $\{x \in A: f(x) \geq \lambda\}$ is a $\tau_{A}$-closed subset of $A$ for all $\lambda \in \mathbb{R}$. We also see that if $f$ is upper semi-continuous on $A$ then $-f$ is lower semi-continuous on $A$, and if $f$ is lower semi-continuous on $A$ then $-f$ is upper semi-continuous on $A$. We have the following observations.

- Suppose that $\tilde{A}$ is a fuzzy set in $U$ such that its membership function $\xi_{\tilde{A}}$ is upper semi-continuous on $U$. Then $\xi_{\tilde{A}}$ is also upper semi-continuous on the proper domain $\tilde{A}_{0}$. Indeed, the set

$$
\left\{x \in \tilde{A}_{0}: \xi_{\tilde{A}}(x) \geq \lambda\right\}=\tilde{A}_{0} \cap\left\{x \in U: \xi_{\tilde{A}}(x) \geq \lambda\right\}
$$

is a $\tau_{\tilde{A}_{0}}$-closed subset of $\tilde{A}_{0}$.

- Suppose that $\tilde{A}$ is a fuzzy set in $U$ such that its membership function $\xi_{\tilde{A}}$ is upper semi-continuous on the proper domain $\tilde{A}_{0}$. Then it is clear to see that the dual membership function $\xi_{\tilde{A}^{\star}}=1-\xi_{\tilde{A}}$ of $\tilde{A}^{\star}$ is lower semi-continuous on $\tilde{A}_{0}$. 
Theorem 4. Let $\tilde{A}$ and $\tilde{B}$ be two fuzzy sets in $\mathbb{R}$ with the dual fuzzy sets $\tilde{A}^{\star}$ and $\tilde{B}^{\star}$, respectively. Suppose that the dual arithmetic operations $\square \in\{\boxplus, \boxminus, \otimes\}$ correspond to the operations $\circ \in\{+,-, *\}$. Then the following statements hold true.

(i) We have $\alpha(\tilde{A} \boxminus \tilde{B})=\varnothing$ for $\alpha \notin I^{\circ, \cap}$.

(ii) We have the inclusion

$$
{ }_{\alpha}(\tilde{A} \boxminus \tilde{B}) \supseteq{ }_{\alpha} \tilde{A}^{\star} \circ{ }_{\alpha} \tilde{B}^{\star}=\tilde{A}_{1-\alpha} \circ \tilde{B}_{1-\alpha} \text { for all } \alpha \in I^{\circ, \cap} .
$$

(iii) Suppose that the membership functions of $\tilde{A}$ and $\tilde{B}$ are upper semi-continuous on $\tilde{A}_{0}$ and $\tilde{B}_{0}$, respectively. Then

$$
{ }_{\alpha}(\tilde{A} \boxminus \tilde{B})={ }_{\alpha} \tilde{A}^{\star} \circ{ }_{\alpha} \tilde{B}^{\star}=\tilde{A}_{1-\alpha} \circ \tilde{B}_{1-\alpha} \text { for all } \alpha \in I^{\circ, \cap} .
$$

Proof. To prove part (i), since $I^{\circ, \cap}=I_{\tilde{A} \boxminus \tilde{B}}^{\circ}$ by (23), Proposition 2 says that $\alpha(\tilde{A} \boxminus \tilde{B})=\varnothing$ for $\alpha \notin I_{\tilde{A} \boxminus \tilde{B}}^{\circ}=I^{\circ, \cap}$.

To prove part (ii), for $\alpha \in I^{\circ, \cap}$ and $z_{\alpha} \in{ }_{\alpha} \tilde{A}^{\star} \circ{ }_{\alpha} \tilde{B}$, there exist $x_{\alpha} \in{ }_{\alpha} \tilde{A}^{\star}$ and $y_{\alpha} \in{ }_{\alpha} \tilde{B}^{\star}$ such that $z_{\alpha}=x_{\alpha} \circ y_{\alpha}$ for $\circ \in\{+,-, *\}$, where $\xi_{\tilde{A}^{\star}}\left(x_{\alpha}\right) \leq \alpha$ and $\xi_{\tilde{B}^{\star}}\left(y_{\alpha}\right) \leq \alpha$. Therefore, we have

$$
\xi_{\tilde{A} \boxminus \tilde{B}}\left(z_{\alpha}\right)=\inf _{\left\{(x, y) \in \tilde{A}_{0} \times \tilde{B}_{0}: z_{\alpha}=x \circ y\right\}} \max \left\{\xi_{\tilde{A}^{\star}}(x), \xi_{\tilde{B}^{\star}}(y)\right\} \leq \max \left\{\xi_{\tilde{A}^{\star}}\left(x_{\alpha}\right), \xi_{\tilde{B}^{\star}}\left(y_{\alpha}\right)\right\} \leq \alpha,
$$

which says that $z_{\alpha} \in{ }_{\alpha}(\tilde{A} \boxminus \tilde{B})$. This shows that ${ }_{\alpha} \tilde{A}^{\star}{ }_{\alpha} \tilde{B}^{\star} \subseteq{ }_{\alpha}(\tilde{A} \boxminus \tilde{B})$ for $\alpha \in I^{\circ}, \cap$.

To prove part (iii), in order to prove another direction of inclusion, we further assume that the membership functions $\xi_{\tilde{A}}$ and $\xi_{\tilde{B}}$ of $\tilde{A}$ and $\tilde{B}$ are upper semi-continuous on $\tilde{A}_{0}$, which imply that the dual membership functions $\xi_{\tilde{A}^{\star}}$ and $\xi_{\tilde{B}^{\star}}$ are lower semi-continuous functions on $\tilde{A}_{0}$; that is, the nonempty lower $\alpha$-level sets ${ }_{\alpha} \tilde{A}^{\star}$ and ${ }_{\alpha} \tilde{B}^{\star}$ are $\tau_{\tilde{A}_{0}}$-closed subsets of $\tilde{A}_{0}$ for all $\alpha \in I^{\circ, \cap}$. Since $_{\alpha}(\tilde{A} \boxminus \tilde{B}) \neq \varnothing$ for $\alpha \in I_{\tilde{A} \sqcup \tilde{B}}^{\circ}=I^{\circ, \cap}$, given any $z_{\alpha} \in{ }_{\alpha}(\tilde{A} \sqcup \tilde{B})$, we have

$$
\inf _{\left\{(x, y) \in \tilde{A}_{0} \times \tilde{B}_{0}: z_{\alpha}=x \circ y\right\}} \max \left\{\xi_{\tilde{A}^{\star}}(x), \xi_{\tilde{B}^{\star}}(y)\right\}=\xi_{\tilde{A} \square \tilde{B}}\left(z_{\alpha}\right) \leq \alpha .
$$

Since $z_{\alpha}$ is finite, it is clear to see that

$$
F \equiv\left\{(x, y) \in \tilde{A}_{0} \times \tilde{B}_{0}: z_{\alpha}=x \circ y\right\}
$$

is a bounded subset of $\tilde{A}_{0} \times \tilde{B}_{0}$. We also see that the function $g(x, y)=x \circ y$ is continuous on $\tilde{A}_{0} \times \tilde{B}_{0}$. Since the singleton set $\left\{z_{\alpha}\right\}$ is a closed subset of $\mathbb{R}$, it follows that the inverse image $F=g^{-1}\left(\left\{z_{\alpha}\right\}\right)$ of $\left\{z_{\alpha}\right\}$ is also a closed subset of $\tilde{A}_{0} \times \tilde{B}_{0}$. This says that $F$ is a compact subset of $\tilde{A}_{0} \times \tilde{B}_{0}$. Now we want to show that the function $f(x, y)=\max \left\{\tilde{\zeta}_{\tilde{A}^{\star}}(x), \xi_{\tilde{B}^{\star}}(y)\right\}$ is lower semi-continuous on $\tilde{A}_{0} \times \tilde{B}_{0}$, i.e., we want to show that

$$
\left\{(x, y) \in \tilde{A}_{0} \times \tilde{B}_{0}: f(x, y) \leq \alpha\right\}
$$

is a closed subset of $\tilde{A}_{0} \times \tilde{B}_{0}$ for any $\alpha \in \mathbb{R}$.

- $\quad$ For $\alpha \in I^{\circ, \cap}=I_{\tilde{A} \boxminus \tilde{B}}^{\circ}=I_{\tilde{A}^{\star}}^{\circ} \cap I_{\tilde{B}^{\star}}^{\circ}$ with $\alpha \geq 0$, i.e., ${ }_{\alpha} \tilde{A}^{\star} \neq \varnothing$ and ${ }_{\alpha} \tilde{B}^{\star} \neq \varnothing$, we have

$$
\begin{aligned}
\left\{(x, y) \in \tilde{A}_{0} \times \tilde{B}_{0}: f(x, y) \leq \alpha\right\} & =\left\{(x, y) \in \tilde{A}_{0} \times \tilde{B}_{0}: \xi_{\tilde{A}^{\star}}(x) \leq \alpha \text { and } \xi_{\tilde{B}^{\star}}(y) \leq \alpha\right\} \\
& =\left\{(x, y) \in \tilde{A}_{0} \times \tilde{B}_{0}: x \in{ }_{\alpha} \tilde{A}^{\star} \text { and } y \in{ }_{\alpha} \tilde{B}^{\star}\right\}={ }_{\alpha} \tilde{A}^{\star} \times{ }_{\alpha} \tilde{B}^{\star}
\end{aligned}
$$

that is a closed subset of $\tilde{A}_{0} \times \tilde{B}_{0}$, since ${ }_{\alpha} \tilde{A}^{\star}$ and ${ }_{\alpha} \tilde{B}^{\star}$ are closed subsets of $\tilde{A}_{0}$ and $\tilde{B}_{0}$, respectively. - If $\alpha<0$, then $\{(x, y): f(x, y) \leq \alpha\}=\varnothing$ is a closed subset of $\tilde{A}_{0} \times \tilde{B}_{0}$. 
- If $\alpha \notin I^{\circ, \cap}$ with $\alpha \geq 0$ then ${ }_{\alpha} \tilde{A}^{\star}=\varnothing$ or ${ }_{\alpha} \tilde{B}^{\star}=\varnothing$. Suppose that ${ }_{\alpha} \tilde{A}^{\star}=\varnothing$. Then $\tilde{\zeta}_{\tilde{A}^{\star}}(x)>\alpha$ for all $x \in \tilde{A}_{0}$. Similarly, if $\alpha \tilde{B}^{\star}=\varnothing$, then $\tilde{\zeta}_{\tilde{B}^{\star}}(y)>\alpha$ for all $y \in \tilde{B}_{0}$. Therefore we conclude that $f(x, y)>\alpha$ for all $(x, y) \in \tilde{A}_{0} \times \tilde{B}_{0}$, which implies

$$
\left\{(x, y) \in \tilde{A}_{0} \times \tilde{B}_{0}: f(x, y) \leq \alpha\right\}=\varnothing
$$

that is also a closed subset of $\tilde{A}_{0} \times \tilde{B}_{0}$.

Therefore the function $f(x, y)$ is indeed lower semi-continuous on $\tilde{A}_{0} \times \tilde{B}_{0}$. By Lemma 2, the function $f$ assumes minimum on the compact subset $F$ of $\tilde{A}_{0} \times \tilde{B}_{0}$; that is, from (24), we have

$$
\min _{(x, y) \in F} f(x, y)=\min _{\left\{(x, y) \in \tilde{A}_{0} \times \tilde{B}_{0}: z_{\alpha}=x \circ y\right\}} f(x, y)=\inf _{\left\{(x, y) \in \tilde{A}_{0} \times \tilde{B}_{0}: z_{\alpha}=x \circ y\right\}} f(x, y) \leq \alpha .
$$

In other words, there exists $\left(x_{\alpha}, y_{\alpha}\right) \in F$ such that $z_{\alpha}=x_{\alpha} \circ y_{\alpha}$ and

$$
\max \left\{\tilde{\zeta}_{\tilde{A}^{\star}}\left(x_{\alpha}\right), \xi_{\tilde{B}^{\star}}\left(y_{\alpha}\right)\right\}=f\left(x_{\alpha}, y_{\alpha}\right)=\min _{(x, y) \in F} f(x, y) \leq \alpha,
$$

i.e., $\xi_{\tilde{A}^{\star}}\left(x_{\alpha}\right) \leq \alpha$ and $\xi_{\tilde{B}^{\star}}\left(y_{\alpha}\right) \leq \alpha$. Therefore, we obtain $x_{\alpha} \in{ }_{\alpha} \tilde{A}^{\star}$ and $y_{\alpha} \in{ }_{\alpha} \tilde{B}^{\star}$, which says that $z_{\alpha} \in{ }_{\alpha} \tilde{A}^{\star} \circ{ }_{\alpha} \tilde{B}^{\star}$, i.e., $\alpha(\tilde{A} \boxminus \tilde{B}) \subseteq{ }_{\alpha} \tilde{A}^{\star} \circ_{\alpha} \tilde{B}^{\star}$ for all $\alpha \in I_{\tilde{A} \square \tilde{B}}^{\circ}=I^{\circ, \cap}$. This completes the proof.

The related results regarding the mixed lower and upper $\alpha$-level sets are presented below. Recall that the 0-level set $(\tilde{A} \odot \tilde{B})_{0}$ is the proper domain of the membership function $\xi_{\tilde{A} \odot \tilde{B}}$ of $\tilde{A} \odot \tilde{B}$.

Theorem 5. Let $\tilde{A}$ and $\tilde{B}$ be two fuzzy sets in $\mathbb{R}$. Consider that the arithmetic operations $\odot \in\{\oplus, \ominus, \otimes\}$ correspond to the operations $\circ \in\{+,-, *\}$. Suppose that the membership functions of $\tilde{A}$ and $\tilde{B}$ are upper semi-continuous. Then we have the following results.

- If $\alpha \geq \min \left\{\sup \mathcal{R}\left(\xi_{\tilde{A}}\right), \sup \mathcal{R}\left(\xi_{\tilde{B}}\right)\right\}$, then ${ }_{\alpha}(\tilde{A} \odot \tilde{B})=(\tilde{A} \odot \tilde{B})_{0}$ that is the proper domain of the membership function $\xi_{\tilde{A} \odot \tilde{B}}$ of $\tilde{A} \odot \tilde{B}$.

- If $0 \leq \alpha<\inf \mathcal{R}\left(\xi_{\tilde{A} \odot \tilde{B}}\right)$, then ${ }_{\alpha}(\tilde{A} \odot \tilde{B})=\varnothing$.

- If $\inf \mathcal{R}\left(\xi_{\tilde{A} \odot \tilde{B}}\right)<\alpha<\min \left\{\sup \mathcal{R}\left(\xi_{\tilde{A}}\right), \sup \mathcal{R}\left(\xi_{\tilde{B}}\right)\right\}$, then

$$
{ }_{\alpha}(\tilde{A} \odot \tilde{B})=(\tilde{A} \odot \tilde{B})_{0} \backslash \bigcup_{\left\{\beta \in I^{*, \cap}: \beta>\alpha\right\}}\left(\tilde{A}_{\beta} \circ \tilde{B}_{\beta}\right)=\bigcup_{\left\{\beta \in I^{*, \cap}: \beta>\alpha\right\}}\left((\tilde{A} \odot \tilde{B})_{0} \backslash\left(\tilde{A}_{\beta} \circ \tilde{B}_{\beta}\right)\right) .
$$

- $\quad$ Let $\alpha=\inf \mathcal{R}\left(\xi_{\tilde{A} \odot \tilde{B}}\right)$. If the minimum $\min \mathcal{R}\left(\xi_{\tilde{A} \odot \tilde{B}}\right)$ does not exist, then ${ }_{\alpha}(\tilde{A} \odot \tilde{B})=\varnothing$. If the minimum $\min \mathcal{R}\left(\xi_{\tilde{A} \odot \tilde{B}}\right)$ exist, then ${ }_{\alpha}(\tilde{A} \odot \tilde{B}) \neq \varnothing$ and can be obtained from (25).

We further assume that the supports $\tilde{A}_{0+}$ and $\tilde{B}_{0+}$ are bounded. Then the above 0-level set $(\tilde{A} \odot \tilde{B})_{0}$ can be replaced by $\tilde{A}_{0} \circ \tilde{B}_{0}$.

Proof. From the first observation of Remark 3, we see that ${ }_{\alpha}(\tilde{A} \odot \tilde{B})=\varnothing$ for $0 \leq \alpha<\inf \mathcal{R}\left(\xi_{\tilde{A} \odot \tilde{B}}\right)$. Also, from (16), if

$$
\alpha \geq \alpha_{\tilde{A} \odot \tilde{B}}^{*} \equiv \sup \mathcal{R}\left(\tilde{\zeta}_{\tilde{A} \odot \tilde{B}}\right)=\min \left\{\sup \mathcal{R}\left(\xi_{\tilde{A}}\right), \sup \mathcal{R}\left(\xi_{\tilde{B}}\right)\right\}
$$


then ${ }_{\alpha}(\tilde{A} \odot \tilde{B})=(\tilde{A} \odot \tilde{B})_{0}$. For $\inf \mathcal{R}\left(\xi_{\tilde{A} \odot \tilde{B}}\right)<\alpha<\alpha_{\tilde{A} \odot \tilde{B}^{\prime}}^{*}$, we have

$$
\begin{aligned}
{ }_{\alpha}(\tilde{A} \odot \tilde{B}) & =(\tilde{A} \odot \tilde{B})_{0} \backslash(\tilde{A} \odot \tilde{B})_{\alpha+} \text { (using the first observation of Remark 3) } \\
& =(\tilde{A} \odot \tilde{B})_{0} \backslash \bigcup_{\alpha<\beta \leq 1}(\tilde{A} \odot \tilde{B})_{\beta} \text { (using part (iv) of Proposition 4) } \\
& =(\tilde{A} \odot \tilde{B})_{0} \backslash \bigcup_{\left\{\beta \in I^{*, \cap}: \beta>\alpha\right\}}\left(\tilde{A}_{\beta} \circ \tilde{B}_{\beta}\right) \text { (using parts (i) and (iii) of Theorem 3). }
\end{aligned}
$$

Finally, using part (iv) of Theorem 3, the 0-level set $(\tilde{A} \odot \tilde{B})_{0}$ can be replaced by $\tilde{A}_{0} \circ \tilde{B}_{0}$. This completes the proof.

Theorem 6. Let $\tilde{A}$ and $\tilde{B}$ be two fuzzy sets in $\mathbb{R}$ with the dual fuzzy set $\tilde{A}^{\star}$ and $\tilde{B}^{\star}$, respectively. Consider the dual arithmetic operations $\square \in\{\boxplus, \boxminus, \otimes\}$ correspond to the operations $\circ \in\{+,-, *\}$. Suppose that the membership functions of $\tilde{A}$ and $\tilde{B}$ are upper semi-continuous on $\tilde{A}_{0}$ and $\tilde{B}_{0}$, respectively. Then we have the following results.

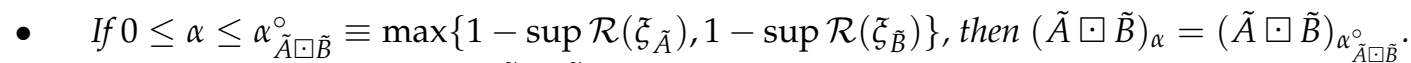

- If $\alpha>\sup \mathcal{R}\left(\tilde{\xi}_{\tilde{A}} \boxminus \tilde{B}\right)$, then $(\tilde{A} \boxminus \tilde{B})_{\alpha}=\varnothing$.

- If $\max \left\{1-\sup \mathcal{R}\left(\xi_{\tilde{A}}\right), 1-\sup \mathcal{R}\left(\xi_{\tilde{B}}\right)\right\}<\alpha<\sup \mathcal{R}\left(\xi_{\tilde{A} \boxminus \tilde{B}}\right)$, then

$$
\begin{aligned}
(\tilde{A} \boxminus \tilde{B})_{\alpha} & =(\tilde{A} \boxminus \tilde{B})_{0} \backslash \bigcup_{\left\{\beta \in I^{\circ, \cap}: 0 \leq \beta<\alpha\right\}}\left({ }_{\beta} \tilde{A}^{\star} \circ_{\beta} \tilde{B}^{\star}\right) \\
& =\bigcup_{\left\{\beta \in I^{\circ, \cap}: 0 \leq \beta<\alpha\right\}}\left((\tilde{A} \boxminus \tilde{B})_{0} \backslash\left({ }_{\beta} \tilde{A}^{\star} \circ_{\beta} \tilde{B}^{\star}\right)\right) .
\end{aligned}
$$

- Let $\alpha=\sup \mathcal{R}\left(\xi_{\tilde{A}} \boxminus \tilde{B}\right)$. If the maximum $\max \mathcal{R}\left(\xi_{\tilde{A}} \boxminus \tilde{B}\right)$ does not exist, then $(\tilde{A} \boxminus \tilde{B})_{\alpha}=\varnothing$. If the maximum $\max \mathcal{R}\left(\tilde{\zeta}_{\tilde{A}} \sqcup \tilde{B}\right)$ exist, then $(\tilde{A} \boxminus \tilde{B})_{\alpha} \neq \varnothing$ and can be obtained from (26).

Proof. From Remark 1, we see that $(\tilde{A} \boxminus \tilde{B})_{\alpha}=\varnothing$ for $\alpha>\sup \mathcal{R}\left(\xi_{\tilde{A}} \boxminus \tilde{B}\right)$. Also, from (17), if

$$
0 \leq \alpha \leq \alpha_{\tilde{A} \boxminus \tilde{B}}^{\circ} \equiv \inf \mathcal{R}\left(\xi_{\tilde{A} \boxminus \tilde{B}}\right)=\max \left\{1-\sup \mathcal{R}\left(\xi_{\tilde{A}}\right), 1-\sup \mathcal{R}\left(\xi_{\tilde{B}}\right)\right\}
$$

then $(\tilde{A} \boxminus \tilde{B})_{\alpha}=(\tilde{A} \sqcup \tilde{B})_{\alpha_{\tilde{A} \square \tilde{B}}^{\circ}}$. For $\alpha_{\tilde{A} \square \tilde{B}}^{\circ}<\alpha<\sup \mathcal{R}\left(\tilde{\xi}_{\tilde{A} \boxminus \tilde{B}}\right)$, we have

$$
\begin{aligned}
(\tilde{A} \boxminus \tilde{B})_{\alpha} & =(\tilde{A} \boxminus \tilde{B})_{0} \backslash \alpha-(\tilde{A} \boxminus \tilde{B}) \text { (using Remark 3) } \\
& =(\tilde{A} \boxminus \tilde{B})_{0} \backslash \bigcup_{0 \leq \beta<\alpha} \beta(\tilde{A} \boxminus \tilde{B}) \text { (using part (iv) of Proposition 3) } \\
& =(\tilde{A} \boxminus \tilde{B})_{0} \backslash \bigcup_{\left\{\beta \in I^{0, \cap}: 0 \leq \beta<\alpha\right\}}\left({ }_{\beta} \tilde{A}^{\star} \circ_{\beta} \tilde{B}^{\star}\right) \text { (using parts (i) and (iii) of Theorem 4). }
\end{aligned}
$$

This completes the proof.

Example 3. Let $\tilde{a}$ and $\tilde{b}$ be two fuzzy numbers. Then we have

$$
\sup \mathcal{R}\left(\xi_{\tilde{a}}\right)=\sup \mathcal{R}\left(\mathcal{\zeta}_{\tilde{b}}\right)=1 \text { and } I^{*, \cap}=[0,1] .
$$

Using Theorem 3, for $\alpha \in[0,1]$, the upper $\alpha$-level set of $\tilde{a} \odot \tilde{b}$ is given by

$$
(\tilde{a} \odot \tilde{b})_{\alpha}=\tilde{a}_{\alpha} \circ \tilde{b}_{\alpha}=\left[\tilde{a}_{\alpha}^{L}, \tilde{a}_{\alpha}^{U}\right] \circ\left[\tilde{b}_{\alpha}^{L}, \tilde{b}_{\alpha}^{U}\right] .
$$


Now we consider the lower $\alpha$-level set of $\tilde{a} \odot \tilde{b}$. From Theorem 5 , the lower 1-level set of $\tilde{a} \odot \tilde{b}$ is given by

$$
{ }_{1}(\tilde{a} \odot \tilde{b})=\tilde{a}_{0} \odot \tilde{b}_{0}=\left[\tilde{a}_{0}^{L}, \tilde{a}_{0}^{U}\right] \circ\left[\tilde{b}_{0}^{L}, \tilde{b}_{0}^{U}\right] .
$$

For $\inf \mathcal{R}\left(\xi_{\tilde{a} \odot \tilde{b}}\right)<\alpha<1$, we have

$$
{ }_{\alpha}(\tilde{a} \odot \tilde{b})=\tilde{a}_{0} \odot \tilde{b}_{0} \backslash \bigcup_{\alpha<\beta \leq 1}\left(\tilde{a}_{\beta} \circ \tilde{b}_{\beta}\right)=\tilde{a}_{0} \odot \tilde{b}_{0} \backslash \bigcup_{\alpha<\beta \leq 1}\left(\left[\tilde{a}_{\beta}^{L}, \tilde{a}_{\beta}^{U}\right] \circ\left[\tilde{b}_{\beta}^{L}, \tilde{b}_{\beta}^{U}\right]\right) .
$$

Suppose that we take $\circ=+$ and $\odot=\oplus$. Then we have

$$
{ }_{1}(\tilde{a} \oplus \tilde{b})=\tilde{a}_{0}+\tilde{b}_{0}=\left[\tilde{a}_{0}^{L}, \tilde{a}_{0}^{U}\right]+\left[\tilde{b}_{0}^{L}, \tilde{b}_{0}^{U}\right]=\left[\tilde{a}_{0}^{L}+\tilde{b}_{0}^{L}, \tilde{a}_{0}^{U}+\tilde{b}_{0}^{U}\right] .
$$

For $\inf \mathcal{R}\left(\xi_{\tilde{a} \oplus \tilde{b}}\right)<\alpha<1$, we have

$$
{ }_{\alpha}(\tilde{a} \oplus \tilde{b})=\left[\tilde{a}_{0}^{L}+\tilde{b}_{0}^{L}, \tilde{a}_{0}^{U}+\tilde{b}_{0}^{U}\right] \backslash \bigcup_{\alpha<\beta \leq 1}\left[\tilde{a}_{\beta}^{L}+\tilde{b}_{\beta}^{L}, \tilde{a}_{\beta}^{U}+\tilde{b}_{\beta}^{U}\right] .
$$

In order to obtain a more simplified form of $\alpha(\tilde{a} \oplus \tilde{b})$, we further assume that the end-points $\tilde{a}_{\alpha}^{L}, \tilde{a}_{\alpha}^{U}, \tilde{b}_{\alpha}^{L}$ and $\tilde{b}_{\alpha}^{U}$ are continuous on $[0,1]$ with respect to $\alpha$. Therefore the endpoints $\tilde{a}_{\beta}^{L}+\tilde{b}_{\beta}^{L}$ and $\tilde{a}_{\beta}^{U}+\tilde{b}_{\beta}^{U}$ are continuous functions on $[0,1]$ with respect to $\beta$, it follows that

$$
\bigcup_{\alpha<\beta \leq 1}\left[\tilde{a}_{\beta}^{L}+\tilde{b}_{\beta}^{L}, \tilde{a}_{\beta}^{U}+\tilde{b}_{\beta}^{U}\right]=\left(\tilde{a}_{\alpha}^{L}+\tilde{b}_{\alpha}^{L}, \tilde{a}_{\alpha}^{U}+\tilde{b}_{\alpha}^{U}\right)
$$

is an open interval. Therefore, for $\inf \mathcal{R}\left(\tilde{\xi}_{\tilde{a} \oplus \tilde{b}}\right)<\alpha<1$, we obtain

$$
{ }_{\alpha}(\tilde{a} \oplus \tilde{b})=\left[\tilde{a}_{0}^{L}+\tilde{b}_{0}^{L}, \tilde{a}_{\alpha}^{L}+\tilde{b}_{\alpha}^{L}\right] \cup\left[\tilde{a}_{\alpha}^{U}+\tilde{b}_{\alpha}^{U}, \tilde{a}_{0}^{U}+\tilde{b}_{0}^{U}\right] .
$$

Example 4. Let $\tilde{a}$ and $\tilde{b}$ be two fuzzy numbers with dual fuzzy set $\tilde{a}^{\star}$ and $\tilde{b}^{\star}$ in $\mathbb{R}$, respectively. According to Example 2 and (23), for $I^{\circ, \cap}=I_{\tilde{a}^{\star}}^{\circ} \cap I_{\tilde{b}^{\star}}^{\circ}$, the lower $\alpha$-level sets of $\tilde{a}^{\star}$ and $\tilde{b}^{\star}$ are nonempty and given by

$$
{ }_{\alpha} \tilde{a}^{\star}=\left[{ }_{\alpha} \tilde{a}^{\star L},{ }_{\alpha} \tilde{a}^{\star U}\right]=\left[\tilde{a}_{1-\alpha}^{L}, \tilde{a}_{1-\alpha}^{U}\right] \text { and }{ }_{\alpha} \tilde{b}^{\star}=\left[{ }_{\alpha} \tilde{b}^{\star L},{ }_{\alpha} \tilde{a}^{\star U}\right]=\left[\tilde{b}_{1-\alpha}^{L}, \tilde{b}_{1-\alpha}^{U}\right] .
$$

Using part (iii) of Theorem 4, the lower $\alpha$-level set of the dual arithmetic $\tilde{a} \square \tilde{b}$ is given by

$$
{ }_{\alpha}(\tilde{a} \sqcup \tilde{b})={ }_{\alpha} \tilde{a}^{\star} \circ{ }_{\alpha} \tilde{b}^{\star}=\left[{ }_{\alpha} \tilde{a}^{\star L},{ }_{\alpha} \tilde{a}^{\star U}\right] \circ\left[{ }_{\alpha} \tilde{b}^{\star L},{ }_{\alpha} \tilde{b}^{\star U}\right]=\left[\tilde{a}_{1-\alpha}^{L}, \tilde{a}_{1-\alpha}^{U}\right] \circ\left[\tilde{b}_{1-\alpha}^{L}, \tilde{b}_{1-\alpha}^{U}\right] .
$$

Using Theorem 6, for $0<\alpha<\sup \mathcal{R}\left(\xi_{\tilde{a} \boxminus \tilde{b}}\right)$, we have

$$
\begin{aligned}
(\tilde{a} \boxminus \tilde{b})_{\alpha} & =(\tilde{a} \boxminus \tilde{b})_{0} \backslash \bigcup_{0 \leq \beta<\alpha}\left({ }_{\beta} \tilde{a}^{\star} \circ{ }_{\beta} \tilde{b}^{\star}\right) \\
& =(\tilde{a} \boxminus \tilde{b})_{0} \backslash \bigcup_{0 \leq \beta<\alpha}\left(\left[{ }_{\beta} \tilde{a}^{\star L}, \beta \tilde{a}^{\star U}\right] \circ\left[{ }_{\beta} \tilde{b}^{\star L},{ }_{\beta} \tilde{b}^{\star U}\right]\right) \\
& =(\tilde{a} \boxminus \tilde{b})_{0} \backslash \bigcup_{0 \leq \beta<\alpha}\left(\left[\tilde{a}_{1-\beta}^{L}, \tilde{a}_{1-\beta}^{U}\right] \circ\left[\tilde{b}_{1-\beta}^{L}, \tilde{b}_{1-\beta}^{U}\right]\right)
\end{aligned}
$$


For $0<\alpha<\sup \mathcal{R}\left(\xi_{\tilde{a} \boxplus \tilde{b}}\right)$, we can similarly show that

$$
\begin{aligned}
\bigcup_{0 \leq \beta<\alpha}\left(\left[\beta \tilde{a}^{\star L}+{ }_{\beta} \tilde{b}^{\star L},{ }_{\beta} \tilde{a}^{\star U}+{ }_{\beta} \tilde{b}^{\star U}\right]\right) & =\left({ }_{\alpha} \tilde{a}^{\star L}+{ }_{\alpha} \tilde{b}^{\star L},{ }_{\alpha} \tilde{a}^{\star U}+{ }_{\alpha} \tilde{b}^{\star U}\right) \\
& =\left(\tilde{a}_{1-\alpha}^{L}+\tilde{b}_{1-\alpha}^{L}, \tilde{a}_{1-\alpha}^{U}+\tilde{b}_{1-\alpha}^{U}\right)
\end{aligned}
$$

is an open interval. Therefore, for $0<\alpha<\sup \mathcal{R}\left(\xi_{\tilde{a} \boxplus \tilde{b}}\right)$, we obtain

$$
(\tilde{a} \boxplus \tilde{b})_{\alpha}=(\tilde{a} \boxplus \tilde{b})_{0} \backslash\left({ }_{\alpha} \tilde{a}^{\star L}+{ }_{\alpha} \tilde{b}^{\star L},{ }_{\alpha} \tilde{a}^{\star U}+{ }_{\alpha} \tilde{b}^{\star U}\right)=(\tilde{a} \boxplus \tilde{b})_{0} \backslash\left(\tilde{a}_{1-\alpha}^{L}+\tilde{b}_{1-\alpha}^{L} \tilde{a}_{1-\alpha}^{U}+\tilde{b}_{1-\alpha}^{U}\right) .
$$

\section{Conclusions}

Let $\tilde{A}$ be a fuzzy subset of a universal set $U$ with membership function denoted by $\xi_{\tilde{A}}$. The conventional $\alpha$-level set is called the upper $\alpha$-level set in this paper. Therefore, we define the so-called lower $\alpha$-level set that is based on the 0-level set $\tilde{A}_{0}$ rather than on the whole universal set $U$. The well-known (primal) decomposition theorem given below

$$
\xi_{\tilde{A}}(x)=\sup _{\alpha \in[0,1]} \alpha \cdot \chi_{\tilde{A}_{\alpha}}(x)=\sup _{\alpha \in(0,1]} \alpha \cdot \chi_{\tilde{A}_{\alpha}}(x)
$$

is based on the normality of $\tilde{A}$. If $\tilde{A}$ is not normal, then we have

$$
\xi_{\tilde{A}}(x)=\sup _{\alpha \in \mathcal{R}\left(\tilde{\zeta}_{\tilde{A}}\right)} \alpha \cdot \chi_{\tilde{A}_{\alpha}}(x) .
$$

The (primal) decomposition theorem says that the membership function can be expressed in terms of upper $\alpha$-level sets. In this paper, we establish the so-called dual decomposition theorem by showing that the membership function can be expressed in terms of lower $\alpha$-level sets as shown below

$$
\begin{aligned}
\xi_{\tilde{A}}(x) & =\sup _{\alpha \in[0,1]} \alpha \cdot \chi_{\alpha \tilde{A}}^{\star}(x)=\max _{\alpha \in[0,1]} \alpha \cdot \chi_{\alpha \tilde{A}}^{\star}(x)=\max _{\alpha \in[0,1]} \alpha \cdot\left[1-\chi_{\alpha}(x)\right] \\
& =\sup _{\alpha \in \mathbb{Q}(0,1]} \alpha \cdot \chi_{\alpha}^{\star} \tilde{A}(x)=\max _{\alpha \in \mathbb{Q}(0,1]} \alpha \cdot \chi_{\alpha}^{\star} \tilde{A}(x)=\max _{\alpha \in \mathbb{Q}(0,1]} \alpha \cdot\left[1-\chi_{\alpha} \tilde{A}(x)\right]
\end{aligned}
$$

by referring to Theorem 2 .

On the other hand, the conventional arithmetic operation between fuzzy sets $\tilde{A}$ and $\tilde{B}$ in $\mathbb{R}$ is defined by

$$
\xi_{\tilde{A} \odot \tilde{B}}(z)=\sup _{\{(x, y) \in U \times U: z=x \circ y\}} \min \left\{\xi_{\tilde{A}}(x), \xi_{\tilde{B}}(y)\right\}
$$

for all $z \in \mathbb{R}$. Using the dual membership functions, the dual arithmetic operation is defined by

$$
\xi_{\tilde{A} \boxminus \tilde{B}}(z)=\inf _{\left\{(x, y) \in \tilde{A}_{0} \times \tilde{B}_{0}: z=x \circ y\right\}} \max \left\{\xi_{\tilde{A}^{\star}}(x), \xi_{\tilde{B}^{\star}}(y)\right\} .
$$

Then the following interesting duality relation is established

$$
\xi_{\tilde{A} \odot \tilde{B}}(z)+\xi_{\tilde{A} \boxminus \tilde{B}}(z)=1 \text { for all } z \in U .
$$

The advantage of considering dual arithmetic is, when $\tilde{A} \boxminus \tilde{B}$ is easier to calculate than that of $\tilde{A} \odot \tilde{B}$, we can just first calculate $\tilde{A} \square \tilde{B}$ and then to recover the arithmetic by using the duality relation.

In future study, we shall apply the dual decomposition theorem and dual arithmetic of fuzzy numbers to investigate the fuzzy real analysis. We also expect to use the results obtained in this paper 
to study the fuzzy problems arisen from the topic of operations research, which are always used to model the engineering problems.

Funding: This research received no external funding.

Conflicts of Interest: The author declares no conflict of interest.

\section{References}

1. Hong, D.H.; Do, H.Y. Additive Decomposition of Fuzzy Quantities. Inf. Sci. 1996, 88, 201-207. [CrossRef]

2. Vrba, J. A Note on Inverses in Arithmetic with Fuzzy Numbers. Fuzzy Sets Syst. 1992, 50, 267-278. [CrossRef]

3. Wu, H.-C. Near Fixed Point Theorems in the Space of Fuzzy Numbers. Mathematics 2018, 6, 108. [CrossRef]

4. Wu, H.-C. Compatibility between Fuzzy Set Operations and Level Set Operations: Applications to Fuzzy Difference. Fuzzy Sets Syst. 2018, 353, 1-43. [CrossRef]

5. Anzilli, L.; Facchinetti, G. New Definitions of Mean Value and Variance of Fuzzy Numbers: An Application to the Pricing of Life Insurance Policies and Real Options. Int. J. Approx. Reason. 2017, 91, 96-113. [CrossRef]

6. Bodjanova, S. Median value and median interval of a fuzzy number. Inf. Sci. 2005, 172, 73-89. [CrossRef]

7. Dubois, D.; Prade, H. The mean value of a fuzzy number. Fuzzy Sets Syst. 1987, 24, 179-300. [CrossRef]

8. Wang, G.; Shi, P.; Xie, Y.; Shi, Y. Two-Dimensional Discrete Fuzzy Numbers and Applications. Inf. Sci. 2016, 32, 258-269. [CrossRef]

9. Mitchell, H.B.; Schaefer, P.A. On Ordering Fuzzy Numbers. Int. J. Intell. Syst. 2000, 15, 981-993. [CrossRef]

10. Yager, R.R.; Filev, D. On Ranking Fuzzy Numbers Using Valuation. Int. J. Intell. Syst. 1999, 14, $1249-1268$. [CrossRef]

11. Deschrijver, G. Arithmetic Operators in Interval-Valued Fuzzy Set Theory. Inf. Sci. 2007, 177, $2906-2924$. [CrossRef]

12. Deschrijver, G. Generalized Arithmetic Operators and Their Relationship to $t$-Norms in Interval-Valued Fuzzy Set Theory. Fuzzy Sets Syst. 2009, 160, 3080-3102. [CrossRef]

13. Ban, A.I.; Coroianu, L. Existence, Uniqueness, Calculus and Properties of Triangular Approximations of Fuzzy Numbers under a General Condition. Int. J. Approx. Reason. 2015, 62, 1-26. [CrossRef]

14. Guerra, M.L.; Stefanini, L. Approximate Fuzzy Arithmetic Operations Using Monotonic Interpolations. Fuzzy Sets Syst. 2005, 150, 5-33. [CrossRef]

15. Holčapek, M.; Štěpnička, M. MI-algebras: A New Frame Work for Arithmetics of (Extensional) Fuzzy Numbers. Fuzzy Sets Syst. 2014, 257, 102-131. [CrossRef]

16. Stupňanová, A. A Probabilistic Approach to the Arithmetics of Fuzzy Numbers. Fuzzy Sets Syst. 2015, 264, 64-75. [CrossRef]

17. Wu, H.-C. Decomposition and Construction of Fuzzy Sets and Their Applications to the Arithmetic Operations on Fuzzy Quantities. Fuzzy Sets Syst. 2013, 233, 1-25. [CrossRef]

18. Royden, H.L. Real Analysis, 2nd ed.; Macmillan: London, UK, 1968.

(C) 2018 by the author. Licensee MDPI, Basel, Switzerland. This article is an open access article distributed under the terms and conditions of the Creative Commons Attribution (CC BY) license (http://creativecommons.org/licenses/by/4.0/). 